\title{
Sharp spectral bounds on starlike domains
}

\author{
Richard S. Laugesen and Bartłomiej A. Siudeja ${ }^{1}$
}

\begin{abstract}
We prove sharp bounds on eigenvalues of the Laplacian that complement the FaberKrahn and Luttinger inequalities. In particular, we prove that the ball maximizes the first eigenvalue and minimizes the spectral zeta function and heat trace. The normalization on the domain incorporates volume and a computable geometric factor that measures the deviation of the domain from roundness, in terms of moment of inertia and a support functional introduced by Pólya and Szegő.

Additional functionals handled by our method include finite sums and products of eigenvalues. The results hold on convex and starlike domains, and for Dirichlet, Neumann or Robin boundary conditions.
\end{abstract}

Mathematics Subject Classification (2010). Primary 35P15; Secondary 35J20, 52A40.

Keywords. Isoperimetric, membrane, spectral zeta, heat trace, partition function, sloshing.

\section{Contents}

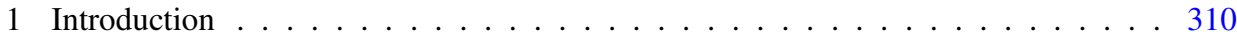

2 The volume preserving transformation, and geometric factors . . . . . . . . 316

3 Main results . . . . . . . . . . . . . . . . . . . . 318

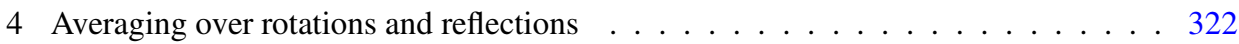

5 Averaging and spherical homeomorphisms . . . . . . . . . . . . 323

6 Dirichlet eigenvalues - Proof of Theorem $3.1 \ldots \ldots \ldots$

7 Neumann eigenvalues - Proof of Theorem $3.3 \ldots \ldots$. . . . . . . . . . 331

8 Robin eigenvalues - Proof of Theorem $3.5 \ldots \ldots \ldots 332$

9 Improvement to the main results $\ldots \ldots \ldots \ldots 33$

10 Properties of the geometric factors . . . . . . . . . . . . . . . . 334

11 Existence of homeomorphism $H$ - Proof of Theorem $2.1 \ldots \ldots$. . . . . . . . . . . . 339

12 Ellipsoidal examples and the geometric factor $G_{1} \ldots \ldots \ldots$. . . . . . . 340

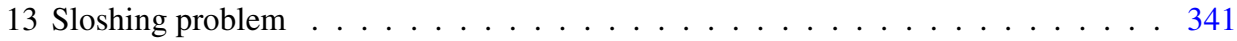

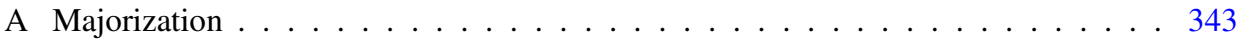

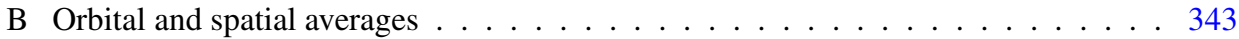

References . . . . . . . . . . . . . . . . . . . . . . . . . 344

\footnotetext{
${ }^{1}$ This work was partially supported by a grant from the Simons Foundation (\#204296 to Richard Laugesen), and travel funding from the University of Oregon.
} 


\section{Introduction}

How do eigenvalues of the Laplacian depend on the shape of the domain? We will obtain new quantitative estimates on the eigenvalues in terms of explicitly computable geometric functionals.

Write $\lambda_{j}$ for the Dirichlet eigenvalues of the Laplacian on the bounded domain $\Omega$ in $\mathbb{R}^{d}, d \geq 2$, with corresponding $L^{2}$-orthonormal eigenfunctions $u_{j}$, so that

$$
\left\{\begin{aligned}
-\Delta u_{j}=\lambda_{j} u_{j} & \text { in } \Omega \\
u_{j}=0 & \text { on } \partial \Omega
\end{aligned}\right.
$$

and

$$
0<\lambda_{1}<\lambda_{2} \leq \lambda_{3} \leq \ldots
$$

These eigenvalues represent physical quantities such as frequencies of vibration, rates of decay to equilibrium in diffusion, and energy levels of quantum particles.

The problem of understanding how eigenvalues are affected by the shape of the domain, and of identifying domains that extremize eigenvalues, is long-standing and difficult. Numerous monographs and survey articles summarize the state of knowledge in euclidean space [3], [4], [7], [19], [23], and [24]. Important results have been obtained on closed surfaces too, for example, see [22], and [39].

Let us first describe our main result in the special case of 2 dimensions (Theorem 1.1). Later we extend to all dimensions (Theorem 3.1) and to Neumann and Robin analogues (Theorem 3.3 and Theorem 3.5), and finally to the sloshing eigenvalues (Section 13).

Consider a starlike domain as in Figure 1 and define scale-invariant geometric factors

$$
G_{0}=\frac{1}{2 \pi} \int_{\partial \Omega} \frac{1}{x \cdot N(x)} d s(x) \text { and } G_{1}=\frac{2 \pi I_{\text {origin }}}{A^{2}},
$$

where $N(x)$ is the outward unit normal vector, $A$ is the area of $\Omega$, and

$$
I_{\text {origin }}=\int_{\Omega}|x|^{2} d A
$$

is the polar moment of inertia about the origin. Note that $x \cdot N(x)>0$ because the domain is starlike. In higher dimensions we will later define $G_{0}$ and $G_{1}$ differently, although the definitions will reduce to (1) in 2 dimensions. 


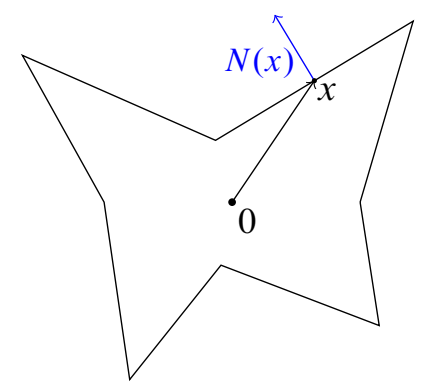

Figure 1. A starlike domain with outer normal $N(x)$.

Let $G=\max \left\{G_{0}, G_{1}\right\}$. Then $G \geq 1$ for all starlike domains with equality for centered disks, by Lemma 2.2 below. Thus one may regard the value of $G$ as measuring the deviation of the domain from roundness. This deviation can arise in two ways: a highly oscillatory starlike boundary would make $G_{0}$ large, while an elongated boundary (such as an eccentric ellipse) would make $G_{1}$ large.

Now we can state the main result in the plane. We show that the disk maximizes eigenvalues of the Laplacian under suitable geometric scaling.

Theorem 1.1 (Dirichlet in 2 dimensions). Suppose the function $R(\theta)$ is $2 \pi$-periodic, positive, and Lipschitz continuous, and consider the starlike domain

$$
\Omega=\left\{r e^{i \theta}: 0 \leq r<R(\theta)\right\} .
$$

Let $n \geq 1$.

Then each of the following scale invariant eigenvalue functionals achieves its maximum value when the domain $\Omega$ is a centered disk:

$$
\lambda_{1} A / G_{0}, \quad \lambda_{2} A / G_{0}, \quad\left(\lambda_{1}^{s}+\cdots+\lambda_{n}^{s}\right)^{1 / s} A / G, \quad \sqrt[n]{\lambda_{1} \lambda_{2} \cdots \lambda_{n}} A / G,
$$

for each exponent $0<s \leq 1$. Further, if $\Phi: \mathbb{R}_{+} \rightarrow \mathbb{R}$ is concave and increasing then $\sum_{j=1}^{n} \Phi\left(\lambda_{j} A / G\right)$ is maximal when $\Omega$ is a disk centered at the origin

Hence the partial sums of the spectral zeta function and trace of the heat kernel are minimal when $\Omega$ is a centered disk. That is, the functionals

$$
\sum_{j=1}^{n}\left(\lambda_{j} A / G\right)^{s} \text { and } \sum_{j=1}^{n} \exp \left(-\lambda_{j} A t / G\right)
$$

attain their smallest value when $\Omega$ is a centered disk, for each $s<0<t$.

The theorem is better for the first and second eigenvalues than for other functionals, in the sense that we normalize $\lambda_{1} A / G_{0}$ and $\lambda_{2} A / G_{0}$ with the quantity $G_{0}$ instead of with $G$, where obviously $G_{0} \leq G$ by definition. 
It is natural in the theorem to multiply $\lambda_{j}$ by $A$, because $\lambda_{j}$ scales like $1 / A$. (Intuitively, low frequencies come from large drums.)

Note the result for $\lambda_{2}$ follows immediately from the one for $\lambda_{1}$, because the ratio $\lambda_{2} / \lambda_{1}$ is maximal on the disk by Ashbaugh and Benguria's "sharp PPW inequality" [2].

The theorem improves on the standard "inradius bounds" for $\lambda_{1}$ and $\lambda_{2}$, on convex domains, as we now show. Write $\mathbb{D}_{i n}$ for the largest open disk centered at the origin and contained in $\Omega$. Then $\lambda_{j}(\Omega) \leq \lambda_{j}\left(\mathbb{D}_{i n}\right)$ for all $j$, by domain monotonicity of the Dirichlet spectrum. Theorem 1.1 implies this inradius bound for $j=1,2$, as one checks by using that $A_{i n} \leq A / G_{0}$ (Lemma 10.2); here $A_{i n}$ is the area of $\mathbb{D}_{i n}$.

Our theorem significantly extends the only known result of its type, which is the case $(n=1)$ of the fundamental tone $\lambda_{1}$ with Dirichlet boundary conditions. That case is due to Pólya and Szegó in 2 dimensions and Freitas and Krejčiřík in higher dimensions, as explained after Theorem 3.1.

To treat higher eigenvalues, we need a fundamentally new idea: we need to transform $\Omega$ into a disk while controlling angular information in the Rayleigh quotients of the eigenfunctions. Any such transformation will change the Rayleigh quotients substantially, and so we must devise a scheme for extracting the geometric effect and leaving behind the portion of the Rayleigh quotient that corresponds to the eigenfunction of the disk.

We construct a geometric transformation that maps linearly on rays and has constant Jacobian. As Figure 2 indicates, wherever the transformation stretches radially it must compress angularly. Section 2 gives the precise definition. We will extract the geometric contribution to the Rayleigh quotient of the trial function on $\Omega$ by composing the transplanted eigenfunction with an arbitrary orthogonal transformation $U$ of the ball and then averaging over all such $U$ (see Proposition 5.2 and Section 6). The constant Jacobian requirement is used here to guarantee that transplanting orthogonal eigenfunctions from the ball will yield orthogonal trial functions on $\Omega$.

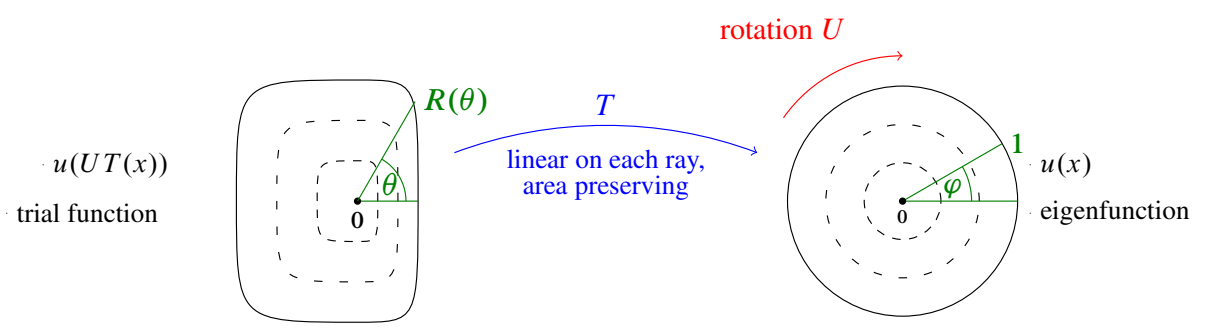

Figure 2. A linear-on-rays transformation from a domain $\Omega$ of area $\pi$ to the unit disk. To insure that the mapping preserves area locally, we require $R(\theta)^{2} d \theta=d \varphi$.

Note that Pólya and Szegő's result on the first eigenvalue was proved by a linearon-rays transformation that does not distort angles and hence does not preserve area. In other words, they took $\theta=\varphi$ in Figure 2. They also did not average over rotations. 
Instead they relied on the special fact that the first Dirichlet eigenfunction of the disk is radial.

Perturbations of the disk. To make the theorem more concrete, we examine the case of nearly circular domains. Suppose $P(\theta)$ is a Lipschitz continuous, $2 \pi$-periodic function. Define a plane domain $\Omega_{\varepsilon}=\left\{r e^{i \theta}: 0 \leq r<1+\varepsilon P(\theta)\right\}$, and assume $\varepsilon$ is small enough that the radius $1+\varepsilon P(\theta)$ is positive for all $\theta$. We may regard $\Omega_{\varepsilon}$ as a perturbation of the unit disk $\mathbb{D}$, since $\Omega_{0}=\mathbb{D}$. Let $j_{0,1} \simeq 2.4$ be the first zero of the Bessel function $J_{0}$, and recall that $\lambda_{1}(\mathbb{D})=j_{0,1}^{2}$.

Corollary 1.2 (Nearly circular domains). The first eigenvalue of the domain $\Omega_{\varepsilon}$ is bounded above and below in terms of the boundary perturbation $P$ :

$$
\begin{aligned}
1 \leq \frac{\lambda_{1}\left(\Omega_{\varepsilon}\right) A\left(\Omega_{\varepsilon}\right)}{j_{0,1}^{2} \pi} & \leq 1+\varepsilon^{2} \int_{0}^{2 \pi} \frac{P^{\prime}(\theta)^{2}}{(1+\varepsilon P(\theta))^{2}} \frac{d \theta}{2 \pi} \\
& =1+\varepsilon^{2}\left(\int_{0}^{2 \pi} P^{\prime}(\theta)^{2} \frac{d \theta}{2 \pi}\right)+O\left(\varepsilon^{3}\right)
\end{aligned}
$$

as $\varepsilon \rightarrow 0$ with $P$ fixed.

Eigenvalue sums satisfy a similar upper bound, for $n \geq 1$ and $s \in(0,1]$ :

$$
\begin{aligned}
& \frac{\left(\sum_{j=1}^{n} \lambda_{j}\left(\Omega_{\varepsilon}\right)^{s}\right)^{1 / s} A\left(\Omega_{\varepsilon}\right)}{\left(\sum_{j=1}^{n} \lambda_{j}(\mathbb{D})^{s}\right)^{1 / s} A(\mathbb{D})} \\
& \quad \leq \max \left\{1+\int_{0}^{2 \pi} \frac{\varepsilon^{2} P^{\prime}(\theta)^{2}}{(1+\varepsilon P(\theta))^{2}} \frac{d \theta}{2 \pi}, \frac{\int_{0}^{2 \pi}(1+\varepsilon P(\theta))^{4} d \theta / 2 \pi}{\left[\int_{0}^{2 \pi}(1+\varepsilon P(\theta))^{2} d \theta / 2 \pi\right]^{2}}\right\} \\
& =1+O\left(\varepsilon^{2}\right) .
\end{aligned}
$$

The lower bound on the first eigenvalue in (2) is the famous Faber-Krahn inequality. The upper bounds are immediate from Theorem 1.1, by taking $R=1+\varepsilon P$ in the formula for $G_{0}$ in Proposition 10.1 and remembering that our two definitions of $G_{0}$ agree in 2 dimensions (Lemma 10.2).

The upper bound on the first eigenvalue in (2) is equivalent to the estimate of Pólya and Szegó [38], pp. 14-15 and 91-92. The upper bound on eigenvalue sums is new.

Let us compare the upper bound on the first eigenvalue with recent work of van den Berg [8], Theorem 1(ii). He obtained an estimate of the form

$$
1+C_{2}\left(\|P\|_{2}\left\|P^{\prime}\right\|_{2}+\|P\|_{2}^{2}\right) \varepsilon^{2}+C_{3}\left\|P^{\prime}\right\|_{2}^{2} \varepsilon^{3},
$$


for perturbations normalized by $\|P\|_{\infty}=1$. Note his formula involves $\|P\|_{2}\left\|P^{\prime}\right\|_{2}$ at second order whereas our corollary has $\left\|P^{\prime}\right\|_{2}^{2}$. When applied to the main example in van den Berg's paper, his estimate beats our estimate (2) by a factor of $\varepsilon^{1 / 2}$ because his $P$ depends on $\varepsilon$ with $\|P\|_{2}=O\left(\varepsilon^{1 / 4}\right)$ and $\left\|P^{\prime}\right\|_{2}=O\left(\varepsilon^{-1 / 4}\right)$. On the other hand, when applied to the "uniform" perturbation $P \equiv-1$, equality holds in our estimate (2) whereas (3) is not exact, due to the contribution of $\|P\|_{2}^{2}$.

Next let us contrast with Rayleigh's second order perturbation expansion of $\lambda_{1}$. See either his formal derivation [40], §210, or Pólya and Szegő's account [38], pp. 132-133, or Henry's more modern approach in all dimensions [18], p. 35. This expansion gives no error bounds, and it holds only for $P$ fixed with $\varepsilon$ tending to 0 . Both our corollary and van den Berg's work establish approximation bounds, and allow $P$ to vary with $\varepsilon$. Rayleigh's perturbation formula is better in one respect, though, because it gives an exact second order term $\left(\varepsilon^{2}\right.$-term) for $\lambda_{1} A$. This term behaves like $\sum|n||\hat{P}(n)|^{2}$, that is, like the square of the $H^{1 / 2}$-norm of the boundary perturbation. In contrast, both our corollary and van den Berg's work have second order terms that are bigger, being controlled by the square of the $H^{1}$-norm, $\sum n^{2}|\widehat{P}(n)|^{2}$.

Prior work, and the new methods. The idea of obtaining eigenvalue bounds by transforming a domain and averaging over rotations appeared already in Laugesen and Morpurgo's 2-dimensional conformal mapping approach [32]. Their averaging task was much easier, though, since it needed only subharmonicity of the modulus of an analytic function. Further, their "reciprocal eigenvalue" results are inherently less powerful than the methods of this paper since, for example, they cannot yield the heat trace for all $t>0$.

More recently, sharp eigenvalue bounds on linear images of rotationally symmetric domains (such as regular polygons) were obtained by the authors and collaborators [30], [31], [33], and [34]. For example, they showed that the centered equilateral triangle maximizes $\left(\lambda_{1}+\cdots+\lambda_{n}\right) A / G_{1}$ among all triangles. The averaging in those papers takes place over discrete groups of rotations (such as 3-fold rotations for triangles), and relies on "tight frame" identities which are special cases of Schur's Lemma from representation theory. The transformations in those papers are globally linear, and so are simpler than the linear-on-rays transformations constructed in this paper. This simplicity comes at the cost of a severely restricted class of image domains.

We must push beyond these existing averaging methods, in this paper. One serious obstacle is the nonlinear nature of our transformation, which causes the rotation matrix $U$ to appear multiple times in the transformed Rayleigh quotient, both inside and outside the derivative of the transformation. We describe how to overcome these obstacles in Section 6.

Faber-Krahn and Luttinger bounds in the reverse direction. Rayleigh conjectured in 1877, and Faber and Krahn proved in the the 1920s, that

$\lambda_{1} A$ is minimal for the disk. 
Many proofs and extensions are known [4], [7], [24], and [38]. The result holds trivially for the first Neumann eigenvalue $\mu_{1}$, which equals zero for each domain. The result holds also for the first Robin eigenvalue, assuming a positive Robin parameter, by work of Bossel and Daners [9], and [13] that was improved to irregular domains by Bucur and Giacomini [11].

This Rayleigh-Faber-Krahn inequality does not extend to sums and products of eigenvalues, as the results in this paper do. It does extend to the spectral zeta function and heat trace, for $s<-1$ and $t>0$ respectively, each of them being maximal for the disk of the same area under Dirichlet boundary conditions. This important extension is due to Luttinger [35], whose multiple integral rearrangement techniques proved remarkably fertile in joint work with Brascamp and Lieb [10]. Note that letting $t \rightarrow \infty$ in the heat trace inequality yields a new proof of the Faber-Krahn theorem.

Luttinger's methods and results do not extend to Neumann boundary conditions. In fact, for large $t$ the area-normalized Neumann heat trace equals approximately $1+e^{-\mu_{2} A t}$, which is minimal for the disk (not maximal) by Szegô-Weinberger's result on the second Neumann eigenvalue [41]. One naturally conjectures that the Neumann heat trace is minimal for the disk of the same area, for each $t>0$. This problem remains open. For the analogous problem of the heat trace on the sphere, Morpurgo [37] has proved local minimality at the round metric.

To put this paper in context, then, one may regard our results as being analogous to the classical Faber-Krahn and Luttinger results except with the direction of their inequalities reversed. Such reversal is made possible by introducing the geometric factor $G$ into the geometric scaling. Further, our theorems improve in three respects on the Faber-Krahn and Luttinger inequalities, because they hold: for finite sums and products of eigenvalues, for each partial sum of the spectral zeta function and heat trace, and for Neumann and Robin boundary conditions in addition to Dirichlet.

Acknowledgments. We thank Michiel van den Berg for asking about perturbations of the ball and alerting us to his recent work [8]. Our Corollary 1.2 and Corollary 3.2 resulted from his question. Thanks go also to Julie Clutterbuck, for suggesting we consider nonconstant Robin parameters in Theorem 3.5. We are grateful to CIRMLuminy and MFO-Oberwolfach for funding the stimulating workshops on "Shape Optimization Problems and Spectral Theory" (May 2012) and "Geometric Aspects of Spectral Theory" (July 2012), respectively, during which these conversations took place.

Lorenzo Brasco pointed out how to deduce the estimate on the second eigenvalue (in Theorem 3.1) from Ashbaugh and Benguria's sharp PPW inequality. The de Giorgi Center at the Scuola Normale in Pisa generously supported our participation in the meeting on "New Trends in Shape Optimization" (July 2012), at which this paper was completed.

Mark Ashbaugh has our gratitude for correcting several mis-statements about the Robin eigenvalues. 


\section{The volume preserving transformation, and geometric factors}

Write $\mathbb{B}=\mathbb{B}^{d}$ for the unit ball centered at the origin, $\mathbb{S}=\mathbb{S}^{d-1}$ for the unit sphere, and $\mathbb{R}_{+}=(0, \infty)$ for the positive half-axis.

We say a domain $\Omega$ in $\mathbb{R}^{d}$ is Lipschitz-starlike if it can be expressed in the form

$$
\Omega=\{r \xi: \xi \in \mathbb{\$}, 0 \leq r<R(\xi)\}
$$

for some positive, Lipschitz continuous function $R(\xi)$ on $\$$. Call $R$ the radius function of $\Omega$. The gauge function is its reciprocal,

$$
\Gamma=\frac{1}{R} .
$$

Write $V$ for volume in $\mathbb{R}^{d}$. Note $V(\Omega)=\frac{1}{d} \int_{\Phi} R(\xi)^{d} d S(\xi)$.

The volume preserving (constant Jacobian) transformation. Our work relies on a map from the Lipschitz-starlike domain $\Omega$ to the ball that preserves volume locally (up to a scale factor), and is linear on each ray from the origin. See Figure 2 for an example in 2 dimensions. First we construct a "boundary homeomorphism" $H$ associated with the radius function $R$ of $\Omega$.

Lemma 2.1. There exists a bi-Lipschitz homeomorphism $H: \mathbb{\$} \rightarrow \mathbb{\$}$ that distorts surface area in proportion to the $d$-th power of the radius function

$$
\operatorname{Jac}_{H}(\xi)=\frac{V(\mathbb{B})}{V(\Omega)} R(\xi)^{d} .
$$

We prove the lemma in Section 11. Simply note at this stage that the left and right sides of (4) both integrate over $\mathbb{\$}$ to yield $|\$|$.

Now define the mapping $T: \Omega \rightarrow \mathbb{B}$ by mapping linearly in each direction and transforming directions with $H$; that is, define

$$
T(r \xi)=\frac{r}{R(\xi)} H(\xi)
$$

for vectors $\xi \in \mathbb{S}$ and numbers $r \in[0, R(\xi))$. One can check that $T$ is a bi-Lipschitz homeomorphism of $\Omega$ to $\mathbb{B}$. Its Jacobian determinant is constant, with

$$
\operatorname{Jac}(T) \equiv V(\mathbb{B}) / V(\Omega) \quad \text { and } \quad \operatorname{Jac}\left(T^{-1}\right) \equiv V(\Omega) / V(\mathbb{B}),
$$

as one deduces from the definition (5) and Jacobian formula (4).

The constant Jacobian property of $T$ will be essential later, when we transplant an orthonormal collection of eigenfunctions on the ball to a collection of functions on $\Omega$. The transplanted functions will remain orthogonal, thanks to the constant Jacobian condition, and so we can use them as trial functions in the Rayleigh principle for the eigenvalue sum. 
The geometric factors. From now on, we extend $R$ and $H$ by homogeneity to be defined not just on the unit sphere but on all nonzero vectors:

$$
R(r \xi)=R(\xi) \text { and } H(r \xi)=H(\xi)
$$

for all $r>0$. Thus it makes sense to speak of the gradient vector $\nabla R$, and the derivative matrix $D H$.

Given a real matrix $M$, write its Hilbert-Schmidt norm as

$$
\|M\|_{H S}=\left(\sum_{j, k} M_{j k}^{2}\right)^{1 / 2}=\left(\operatorname{tr} M^{\dagger} M\right)^{1 / 2},
$$

where $M^{\dagger}$ denotes the transposed matrix. All matrices in this paper will be real.

Now define geometric quantities

$$
G_{0}(\Omega)=\frac{\frac{1}{|\mathbb{S}|} \int_{\mathbb{S}}\left[R(\xi)^{d-2}+|\nabla R(\xi)|^{2} R(\xi)^{d-4}\right] d S(\xi)}{\left(\frac{1}{|\mathbb{}|} \int_{\Phi} R(\xi)^{d} d S(\xi)\right)^{(d-2) / d}}
$$

and

$$
G_{1}(\Omega)=\frac{\frac{1}{|\mathbb{S}|} \int_{\Phi} \frac{\|D H(\xi)\|_{H S}^{2}}{d-1} R(\xi)^{d-2} d S(\xi)}{\left(\frac{1}{|\Phi|} \int_{\Phi} R(\xi)^{d} d S(\xi)\right)^{(d-2) / d}} .
$$

Clearly $G_{0}$ and $G_{1}$ are scale invariant, meaning that $G_{i}(\Omega)=G_{i}(a \Omega)$ for all $a>0$, since $a \Omega$ has radius function $a R$.

Alternative formulas for $G_{0}$ and $G_{1}$ of a geometric nature will be developed in Section 10 . There we express $G_{0}$ in terms of a support-type integral over the boundary that was employed previously by Pólya and Szegó, and we show in two dimensions that $G_{1}=2 \pi I_{\text {origin }} / A^{2}$. Thus these alternative formulas recover the definitions (1) that we used in the plane, and show that in 2 dimensions, both $G_{0}$ and $G_{1}$ depend only on the shape of $\Omega$ and on the choice of origin.

In higher dimensions, $G_{1}$ depends also on the choice of homeomorphism $H$.

Example. If $\Omega$ is a centered ball one has $R \equiv$ const., so that $G_{0}=1$. By convention, for a centered ball we choose the homeomorphism $H$ to be the identity on the sphere, so that $G_{1}=1$.

Lemma 2.2. The geometric quantities are always at least 1 in value:

$$
G_{0} \geq 1 \text { and } G_{1} \geq 1 .
$$

Equality statement: $G_{0}=1$ if and only if $\Omega$ is a centered ball, and $G_{1}=1$ if and only if $\Omega$ is a centered ball and $H$ is an orthogonal transformation of the sphere. 
This lemma helps us interpret the main theorem below. We do not otherwise need the lemma though, and so we defer its proof to Section 10.

\section{Main results}

First we extend the eigenvalues estimates in Theorem 1.1 to all dimensions. Let

$$
G=\max \left\{G_{0}, G_{1}\right\} .
$$

Theorem 3.1 (Dirichlet). Assume $\Omega$ is a Lipschitz-starlike domain in $\mathbb{R}^{d}, d \geq 2$, and let $n \geq 1$. Then the scale invariant eigenvalue functionals

$\lambda_{1} V^{2 / d} / G_{0}, \quad \lambda_{2} V^{2 / d} / G_{0}, \quad\left(\lambda_{1}^{s}+\cdots+\lambda_{n}^{s}\right)^{1 / s} V^{2 / d} / G, \quad \sqrt[n]{\lambda_{1} \lambda_{2} \cdots \lambda_{n}} V^{2 / d} / G$,

are maximal when $\Omega$ is a centered ball, for each exponent $0<s \leq 1$. Further, if $\Phi: \mathbb{R}_{+} \rightarrow \mathbb{R}$ is concave and increasing then $\sum_{j=1}^{n} \Phi\left(\lambda_{j} V^{2 / d} / G\right)$ is maximal when $\Omega$ is a centered ball. Hence for $s<0<t$ the functionals

$$
\sum_{j=1}^{n}\left(\lambda_{j} V^{2 / d} / G\right)^{s} \text { and } \sum_{j=1}^{n} \exp \left(-\lambda_{j} V^{2 / d} t / G\right)
$$

are minimal when $\Omega$ is a centered ball.

Equality statement for the first eigenvalue: if $\lambda_{1} V^{2 / d} /\left.G_{0}\right|_{\Omega}=\lambda_{1} V^{2 / d} /\left.G_{0}\right|_{\mathbb{B}}$ and $R$ is $C^{2}$-smooth then $\Omega$ is a centered ball.

The proof is in Section 6.

The only part of the theorem known previously was the estimate on the first eigenvalue. This extremal result for $\lambda_{1} V^{2 / d} / G_{0}$ was proved by Pólya and Szegô [38], pp. 14-15 and 91-92, in 2 dimensions, and by Freitas and Krejčiřík [16], Theorem 3, in higher dimensions. The geometric factors in those papers look different from our $G_{0}$, but they are equivalent, as we explain in Section 10 .

Note that for the first (and second) eigenvalue, the conclusion of our theorem is stronger than for the general case, because it uses $G_{0}$ instead of $G$. The underlying reason is that the first eigenfunction of a ball is purely radial, so that our proof does not depend on the angular information encoded in the homeomorphism $H$ and factor $G_{1}$.

We strengthen the theorem in Section 9 by adapting the geometric factor to each eigenvalue. There we replace $G$ with a convex combination of $G_{0}$ and $G_{1}$ (rather than their maximum).

Perturbations of the ball. Let us see what Theorem 3.1 says for nearly spherical domains. Suppose $P(\xi)$ is a Lipschitz continuous function on the sphere $\mathbb{\$}$, and define a domain $\Omega_{\varepsilon}=\{r \xi: 0 \leq r<1+\varepsilon P(\xi)\}$, assuming $0<1+\varepsilon P(\xi)$ for all $\xi$. 
Corollary 3.2 (Nearly spherical domains). The first eigenvalue of the domain $\Omega_{\varepsilon}$ can be bounded above and below in terms of the radial perturbation $P$ :

$$
\begin{aligned}
1 & \leq \frac{\lambda_{1}\left(\Omega_{\varepsilon}\right) V\left(\Omega_{\varepsilon}\right)^{2 / d}}{\lambda_{1}(\mathbb{B}) V(\mathbb{B})^{2 / d}} \\
& \leq G_{0}\left(\Omega_{\varepsilon}\right) \\
& =1+\left(\int_{\mathbb{S}}|\nabla P|^{2} \frac{d S}{|\mathbb{S}|}-(d-2) \int_{\mathbb{S}}(P-\bar{P})^{2} \frac{d S}{|\mathbb{S}|}\right) \varepsilon^{2}+O\left(\varepsilon^{3}\right)
\end{aligned}
$$

as $\varepsilon \rightarrow 0$ with $P$ fixed, where

$$
\bar{P}=\int_{\Phi} P d S /|\$|
$$

is the mean value of the perturbation.

The lower bound is simply the Faber-Krahn result. The upper bound appears not to have been stated before. It follows by straightforward calculations from Theorem 3.1, simply substituting $R=1+\varepsilon P$ into the definition (6) of $G_{0}$. It can be compared with van den Berg's result [8], Theorem 1(ii), just like in 2 dimensions — see the remarks after Corollary 1.2.

Amusingly, the corollary implies a Poincaré inequality on the sphere, since the $\varepsilon^{2}$-term is necessarily nonnegative.

Neumann and Robin boundary conditions. Denote the Neumann eigenvalues by $\mu_{j}$, assuming that $\partial \Omega$ is Lipschitz so that the spectrum exists and is discrete. Write $u_{j}$ for corresponding orthonormal eigenfunctions. Then

$$
\left\{\begin{aligned}
-\Delta u_{j}=\mu_{j} u_{j} & \text { in } \Omega \\
\frac{\partial u_{j}}{\partial n}=0 & \text { on } \partial \Omega
\end{aligned}\right.
$$

and

$$
0=\mu_{1}<\mu_{2} \leq \mu_{3} \leq \ldots .
$$

We will ignore the first eigenvalue, in the next theorem, since $\mu_{1}=0$ for every domain.

Theorem 3.3 (Neumann). Assume $\Omega$ is a Lipschitz-starlike domain in $\mathbb{R}^{d}, d \geq 2$. Suppose $\Phi: \mathbb{R}_{+} \rightarrow \mathbb{R}$ is concave and increasing, and let $n \geq 2$. Then the scale invariant eigenvalue functional $\sum_{j=2}^{n} \Phi\left(\mu_{j} V^{2 / d} / G\right)$ is maximal when $\Omega$ is a centered ball.

In particular, for $0<s \leq 1$ the functionals

$$
\mu_{2} V^{2 / d} / G, \quad\left(\mu_{2}^{s}+\cdots+\mu_{n}^{s}\right)^{1 / s} V^{2 / d} / G, \quad \sqrt[n-1]{\mu_{2} \cdots \mu_{n}} V^{2 / d} / G,
$$


are maximal when $\Omega$ is a centered ball. For $s<0<t$ the functionals

$$
\sum_{j=2}^{n}\left(\mu_{j} V^{2 / d} / G\right)^{s} \text { and } \sum_{j=2}^{n} \exp \left(-\mu_{j} V^{2 / d} t / G\right)
$$

are minimal when $\Omega$ is a centered ball.

Equality statement for the first nonzero eigenvalue: if $\mathrm{R}$ and $\mathrm{H}$ are $\mathrm{C}^{2}$-smooth and $\mu_{2} V^{2 / d} /\left.G\right|_{\Omega}=\mu_{2} V^{2 / d} /\left.G\right|_{\mathbb{B}}$ then $\Omega$ is a centered ball.

Section 7 has the proof. Because $G \geq 1$, the bound on $\mu_{2} V^{2 / d} / G$ in Theorem 3.3 follows from the Szegó-Weinberger theorem that $\mu_{2} V^{2 / d}$ is maximal for the ball (see [41] or [19], Theorem 7.1.1). Note that Theorem 3.3 holds for higher eigenvalue functionals too, which the Szegó-Weinberger theorem does not.

Theorem 3.3 might hold with $G$ replaced by the smaller quantity $G_{1}$ (the moment of inertia type functional), as we have conjectured elsewhere [33], §4.

Next we turn to Robin boundary conditions. The Robin eigenvalue problem is

$$
\begin{cases}-\hbar^{2} \Delta u_{j}=\rho_{j} u_{j} & \text { in } \Omega, \\ \hbar^{2} \frac{\partial u_{j}}{\partial n}+\sigma u_{j}=0 & \text { on } \partial \Omega,\end{cases}
$$

with eigenvalues

$$
\rho_{1}<\rho_{2} \leq \rho_{3} \leq \ldots,
$$

where $\sigma \in L^{\infty}(\partial \Omega)$ is the Robin parameter and $\hbar>0$ is the Planck constant. Existence and discreteness of the Robin spectrum under these assumptions follows from the usual quadratic form approach; see for example [29], Chapter 5. Note the Robin parameter $\sigma$ will be fixed in our work. For interesting asymptotic behavior of Robin eigenvalues as $\sigma$ varies and approaches $\pm \infty$, see [14] and [25] and references therein.

The Robin eigenvalues reduce to Neumann when $\hbar=1, \sigma \equiv 0$.

In the Dirichlet and Neumann eigenvalue problems we took $\hbar=1$. That causes no loss of generality, since one can always adjust the value of $\hbar$ by rescaling the domain. In our Robin result below, though, the Planck constant and Robin parameter will be multiplied by different geometric factors. Accordingly we write $\rho_{j}=\rho_{j}(\Omega, \hbar, \sigma)$ to display the dependence of the $j$ th Robin eigenvalue on the domain, Planck constant and Robin parameter.

Our theorem will involve a new geometric factor,

$$
G_{\text {Robin }}=\left(\frac{|\partial \Omega| / V(\Omega)^{(d-1) / d}}{|\partial \mathbb{B}| / V(\mathbb{B})^{(d-1) / d}}\right)^{2} .
$$

Clearly $G_{\text {Robin }} \geq 1$ by the isoperimetric inequality, with equality if and only if $\Omega$ is a ball. And $G_{0}$ is larger than $G_{\text {Robin }}$. 
Lemma 3.4. $G_{0} \geq G_{\text {Robin }} \geq 1$.

The lemma was proved in 2 dimensions by Aissen [1], Theorem 1. Our proof appears in Section 10, and is valid in all dimensions.

Now we can state our sharp upper bound on Robin eigenvalues.

Theorem 3.5 (Robin). Assume $\Omega$ is a Lipschitz-starlike domain in $\mathbb{R}^{d}, d \geq 2$. Suppose $\Phi: \mathbb{R} \rightarrow \mathbb{R}$ is concave and increasing, and let $n \geq 1$. Then

$$
\sum_{j=1}^{n} \Phi\left(\rho_{j}\left(\Omega, \hbar V^{1 / d} / G^{1 / 2}, \sigma V^{1 / d} / G_{\text {Robin }}^{1 / 2}\right)\right)
$$

is maximal when $\Omega$ is a centered ball and $\sigma$ is replaced by its average value.

For the first eigenvalue one has a stronger result (with $G_{0}$ instead of $G$ ):

$$
\rho_{1}\left(\Omega, \hbar \bar{R} / G_{0}^{1 / 2}, \sigma \bar{R} / G_{\text {Robin }}^{1 / 2}\right) \leq \rho_{1}(\mathbb{B}, \hbar, \bar{\sigma})
$$

where $\bar{R}$ is the radius of a ball having the same volume as $\Omega$ and

$$
\bar{\sigma}=\int_{\partial \Omega} \sigma d S /|\partial \Omega|
$$

is the average value of the Robin parameter. If equality holds in (9) and if $R$ is $C^{2}$-smooth, then $\Omega$ is a centered ball.

See Section 8 for the proof.

Note that if $\sigma>0$ then the Robin eigenvalues are all positive, in which case $\Phi$ need only be concave and increasing on the half-axis $\mathbb{R}_{+}$.

A particularly simple corollary holds for the ball: averaging the Robin parameter increases the eigenvalue functionals on a ball, with

$$
\sum_{j=1}^{n} \Phi\left(\rho_{j}(\mathbb{B}, \hbar, \sigma)\right) \leq \sum_{j=1}^{n} \Phi\left(\rho_{j}(\mathbb{B}, \hbar, \bar{\sigma})\right) .
$$

Remark. Bareket [6], Appendix A, applied the Pólya-Szegő trial function technique with a constant parameter $\sigma<0$ and got an upper bound on $\rho_{1}$. She did not attach geometric factors to the Planck constant or Robin parameter, though, and so her bound looks different from ours in (9).

Bareket raised an analogue of Rayleigh's Conjecture for negative Robin parameter, namely that

$$
\rho_{1}(\Omega, 1, \bar{\sigma}) \leq \rho_{1}(\mathbb{B}, 1, \bar{\sigma}),
$$


whenever $\bar{\sigma}<0$ is constant and $\Omega$ has the same volume as $\mathbb{B}$. Note the ball is the maximizer here, which is the opposite of the Faber-Krahn type result by Bossel and Daners that holds when $\bar{\sigma} \geq 0$. Let us compare Bareket's conjecture with our result (9) for the first eigenvalue, which says (when $\hbar=1, \bar{R}=1$ and $\sigma \equiv \bar{\sigma}<0$ ) that

$$
\rho_{1}\left(\Omega, 1 / G_{0}^{1 / 2}, \sigma / G_{\text {Robin }}^{1 / 2}\right) \leq \rho_{1}(\mathbb{B}, 1, \bar{\sigma}) .
$$

The factors $G_{0}$ and $G_{\text {Robin }}$ are both greater than 1 , and so the Planck constant and Robin parameter are smaller in magnitude on the left side of our inequality than on the left side of Bareket's conjecture. In particular, our Robin parameter is less negative than Bareket's. Thus while our Planck constant tends to make the Rayleigh quotient smaller than it would be with Bareket's Planck constant, our Robin parameter tends to make it bigger. Hence our result is most likely not comparable to her conjecture.

\section{Averaging over rotations and reflections}

Our proofs will involve averaging over the group of all orthogonal transformations.

Write $\gamma=\gamma_{d}$ for the Haar probability measure on the group $O(d)$ of orthogonal, real, $d \times d$ matrices. Let "Id" denote the identity matrix.

Lemma 4.1 (Averaging in a conjugacy class). Suppose $M$ is a $d \times d$ real symmetric matrix, for some $d \geq 1$. Then

$$
\int_{O(d)} U^{-1} M U d \gamma(U)=\frac{1}{d} \operatorname{tr}(M) \operatorname{Id} .
$$

Hence for each column vector $m \in \mathbb{R}^{d}$,

$$
\int_{O(d)}\left(\begin{array}{cc}
U & 0 \\
0 & 1
\end{array}\right)^{-1}\left(\begin{array}{cc}
M & m \\
m^{\dagger} & 0
\end{array}\right)\left(\begin{array}{cc}
U & 0 \\
0 & 1
\end{array}\right) d \gamma(U)=\frac{1}{d} \operatorname{tr}(M)\left(\begin{array}{cc}
\mathrm{Id} & 0 \\
0 & 0
\end{array}\right) .
$$

The lemma is a special case of Schur's Lemma from representation theory. We give a short proof, for the sake of completeness.

Proof of Lemma 4.1. Denote the left side of formula (10) by

$$
L=\int_{O(d)} U^{-1} M U d \gamma(U) .
$$

For any $U \in O(d)$, we have $L U=U L$ by invariance of Haar measure. Let $\alpha$ be a real eigenvalue of $L$ with eigenvector $w \in \mathbb{R}^{d}$ (using here that $L$ is symmetric, by symmetry of $M$ and orthogonality of $U$ ). Then

$$
L(U w)=U(L w)=U(\alpha w)=\alpha(U w) .
$$


Hence each vector in the orbit $\{U w: U \in O(d)\}$ is an eigenvector of $L$ with eigenvalue $\alpha$. The orbit spans all of $\mathbb{R}^{d}$, and so $L$ equals $\alpha$ times the identity. Taking the trace yields

$$
\alpha d=\operatorname{tr} L=\int_{O(d)} \operatorname{tr}\left(U^{-1} M U\right) d \gamma(U)=\int_{O(d)} \operatorname{tr}(M) d \gamma(U)=\operatorname{tr}(M) .
$$

Hence $L$ equals $\frac{1}{d} \operatorname{tr}(M)$ times the identity.

The second formula in the lemma follows immediately, by multiplying out the matrices and using that

$$
\int_{O(d)} U d \gamma(U)=0
$$

\section{Averaging and spherical homeomorphisms}

Consider a bi-Lipschitz homeomorphism $H: \mathbb{\$} \rightarrow \mathbb{\$}$, extended by homogeneity to $\mathbb{R}^{d} \backslash\{0\}$ so that $H(r \xi)=H(\xi)$ for all $r>0, \xi \in \mathbb{}$.

Lemma 5.1 (Orthogonality relation). For all $\xi \in \mathbb{S}$, we have $(D H)^{\dagger}(\xi) H(\xi)=0$.

Proof of Lemma 5.1. We have $|H(x)|^{2} \equiv 1$ for all $x \neq 0$, because $H$ takes values in the unit sphere. Taking the gradient of this last identity yields that $2 H(x)^{\dagger} D H(x) \equiv$ 0 . Applying the transpose and evaluating at $x=\xi$ completes the proof.

The lemma implies that $D H(\xi) y \cdot H(\xi)=0$ for all vectors $y$, so that the range of the derivative operator $D H$ at $\xi$ lies orthogonal to the image vector $H(\xi)$, as one would expect since $H$ maps into a sphere.

Some complicated expressions involving $H$ can be simplified considerably, after we average over all orthogonal matrices $U$. We will encounter expressions of the following type when we prove our main theorem.

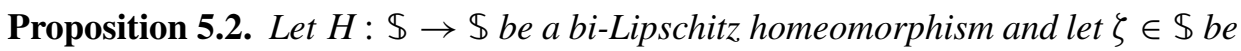
a fixed unit vector.

If $F \in L^{\infty}\left(\mathbb{S} ; \mathbb{R}^{d}\right)$ is a bounded, row-vector valued function on the sphere, then

$$
\int_{O(d)} F\left(H^{-1}(U \zeta)\right)(D H)^{\dagger}\left(H^{-1}(U \zeta)\right) U d \gamma(U)=0 .
$$

If $f \in L^{\infty}(\mathbb{S} ; \mathbb{R})$ is a bounded, real-valued function on the sphere, then

$$
\begin{aligned}
& \int_{O(d)} f\left(H^{-1}(U \zeta)\right) U^{-1} D H\left(H^{-1}(U \zeta)\right)(D H)^{\dagger}\left(H^{-1}(U \zeta)\right) U d \gamma(U) \\
& \quad=c\left(\mathrm{Id}-\zeta \zeta^{\dagger}\right)
\end{aligned}
$$


where

$$
c=\frac{1}{|\mathbb{S}|} \int_{\mathbb{S}} f(\xi) \frac{\|D H(\xi)\|_{H S}^{2}}{d-1} \operatorname{Jac}_{H}(\xi) d S(\xi) .
$$

Proof of Proposition 5.2. Choose an orthogonal matrix $W$ that maps $\zeta$ to the north pole, meaning $W \zeta=v$ where $v=(0, \ldots, 0,1)^{\dagger}$ is the north pole column vector.

We have that

$$
\begin{aligned}
& \int_{O(d)} F\left(H^{-1}(U \zeta)\right)(D H)^{\dagger}\left(H^{-1}(U \zeta)\right) U d \gamma(U) \\
& =\int_{O(d)} F\left(H^{-1}(U v)\right)(D H)^{\dagger}\left(H^{-1}(U \nu)\right) U V W d \gamma(U)
\end{aligned}
$$

by changing variable with $U \mapsto U V W$, where $V$ is an arbitrary matrix in $O(d)$ that fixes the north pole (that is, $V v=v$ ). We may write

$$
V=\left(\begin{array}{ll}
\tilde{V} & 0 \\
0 & 1
\end{array}\right)
$$

where $\tilde{V} \in O(d-1)$.

We are permitted to average expression (12) with respect to $\tilde{V}$, since formula (11) does not depend on $\widetilde{V}$. Note $V$ appears only once in (12). Averaging it yields

$$
\int_{O(d-1)} V d \gamma_{d-1}(\tilde{V})=\left(\begin{array}{ll}
0 & 0 \\
0 & 1
\end{array}\right)=v v^{\dagger}
$$

Multiplying on the right by $W$ gives

$$
\int_{O(d-1)} V W d \gamma_{d-1}(\tilde{V})=v \zeta^{\dagger}
$$

because $W \zeta=v$ by construction. Hence after averaging (12) with respect to $\tilde{V}$ we obtain that expression (11) equals

$$
\int_{O(d)} F\left(H^{-1}(U v)\right)(D H)^{\dagger}\left(H^{-1}(U v)\right) U v \zeta^{\dagger} d \gamma(U)=0
$$

because

$$
(D H)^{\dagger}\left(H^{-1}(U v)\right) U v=0
$$

by Lemma 5.1 applied with $\xi=H^{-1}(U v)$.

Now we prove the second formula in the proposition. We find

$$
\begin{aligned}
& \int_{O(d)} f\left(H^{-1}(U \zeta)\right) U^{-1} D H\left(H^{-1}(U \zeta)\right)(D H)^{\dagger}\left(H^{-1}(U \zeta)\right) U d \gamma(U) \\
& =\int_{O(d)} f\left(H^{-1}(U v)\right) W^{-1}\left(V^{-1} M V\right) W d \gamma(U)
\end{aligned}
$$


by changing variable with $U \mapsto U V W$ as before, where we have defined a matrix

$$
M=U^{-1} D H\left(H^{-1}(U v)\right)(D H)^{\dagger}\left(H^{-1}(U v)\right) U .
$$

Note that $M v=0$ by (14), and similarly $v^{\dagger} M=0$ by symmetry of $M$. Hence $M$ has the form

$$
M=\left(\begin{array}{cc}
\tilde{M} & 0 \\
0 & 0
\end{array}\right) .
$$

Thus using the decomposition (13) for $V$, we conclude from Lemma 4.1 that

$$
\int_{O(d-1)} V^{-1} M V d \gamma_{d-1}(\tilde{V})=\frac{1}{d-1}(\operatorname{tr} \tilde{M})\left(\begin{array}{cc}
\operatorname{Id}_{d-1} & 0 \\
0 & 0
\end{array}\right) .
$$

Note formula (15) does not depend on $V$. Thus after averaging expression (16) with respect to $\widetilde{V}$ and using the last formula, we find that expression (15) equals

$$
\tilde{c} W^{-1}\left(\begin{array}{cc}
\operatorname{Id}_{d-1} & 0 \\
0 & 0
\end{array}\right) W
$$

where

$$
\tilde{c}=\frac{1}{d-1} \int_{O(d)} f\left(H^{-1}(U v)\right)(\operatorname{tr} \tilde{M}) d \gamma(U)
$$

Since

$$
W^{-1}\left(\begin{array}{cc}
\operatorname{Id}_{d-1} & 0 \\
0 & 0
\end{array}\right) W=W^{-1}\left(\mathrm{Id}-v v^{\dagger}\right) W=\mathrm{Id}-\zeta \zeta^{\dagger},
$$

we deduce that expression (15) equals $\tilde{c}\left(\mathrm{Id}-\zeta \zeta^{\dagger}\right)$.

Note that

$$
\begin{aligned}
\operatorname{tr} \tilde{M}=\operatorname{tr} M & =\operatorname{tr}\left(D H\left(H^{-1}(U v)\right)(D H)^{\dagger}\left(H^{-1}(U v)\right)\right) \\
& =\left\|D H\left(H^{-1}(U v)\right)\right\|_{H S}^{2} .
\end{aligned}
$$

Hence $\tilde{c}$ can be evaluated by using the equivalence between orbital and spatial averages (Appendix B), which gives that

$$
\tilde{c}=\frac{1}{d-1} \frac{1}{|\mathbb{S}|} \int_{\Phi} f\left(H^{-1}\left(\zeta^{\prime}\right)\right)\left\|D H\left(H^{-1}\left(\zeta^{\prime}\right)\right)\right\|_{H S}^{2} d S\left(\zeta^{\prime}\right) .
$$

Lastly, changing variable with $\zeta^{\prime}=H(\xi)$ shows that $\tilde{c}$ equals the constant $c$ defined in the Proposition.

\section{Dirichlet eigenvalues - Proof of Theorem 3.1}

The idea is to obtain trial functions on $\Omega$ by transplanting eigenfunctions from $\mathbb{B}$ to $\Omega$ with the volume-preserving map $T$, and then to average with respect to rotations and reflections of $\mathbb{B}$. 
Recall that the Rayleigh quotient associated with the Dirichlet spectrum is

$$
\operatorname{Ray}[u]=\frac{\int_{\Omega}|\nabla u|^{2} d x}{\int_{\Omega} u^{2} d x}, \quad u \in H_{0}^{1}(\Omega) .
$$

The Rayleigh-Poincaré Variational Principle [4], p. 98, characterizes the sum of the first $n$ Dirichlet eigenvalues as

$$
\begin{aligned}
\lambda_{1}+\cdots+ & \lambda_{n} \\
=\min \{ & \operatorname{Ray}\left[v_{1}\right]+\cdots+\operatorname{Ray}\left[v_{n}\right]: \\
& \left.v_{1}, \ldots, v_{n} \in H_{0}^{1}(\Omega) \text { are pairwise orthogonal in } L^{2}(\Omega)\right\} .
\end{aligned}
$$

To use this principle, let $u_{1}, u_{2}, u_{3}, \ldots$ be orthonormal eigenfunctions on $\mathbb{B}$ corresponding to the eigenvalues $\lambda_{1}(\mathbb{B}), \lambda_{2}(\mathbb{B}), \lambda_{3}(\mathbb{B}), \ldots$ Take an orthogonal matrix $U$. Then define trial functions

$$
v_{j}=u_{j} \circ U^{-1} \circ T
$$

on the domain $\Omega$, where the transformation $T: \Omega \rightarrow \mathbb{B}$ was defined in Section 2 . Clearly $v_{j} \in L^{2}(\Omega)$ since $T$ has constant Jacobian. One can further show that $v_{j}$ has weak derivatives in $L^{2}(\Omega)$, since $u_{j}$ is smooth with derivatives in $L^{2}(\mathbb{B})$ and the Lipschitz continuous mapping $T$ has bounded weak derivatives. Thus $v_{j} \in H^{1}(\Omega)$. Further, $v_{j}=0$ on $\partial \Omega$ because $u_{j}=0$ on $\partial \mathbb{B}$; more precisely, $v_{j} \in H_{0}^{1}(\Omega)$ because $u_{j} \in H_{0}^{1}(\mathbb{B})$.

The functions $v_{j}$ are pairwise orthogonal, since

$$
\int_{\Omega} v_{j} v_{k} d x=\operatorname{Jac}\left(T^{-1}\right) \int_{\mathbb{B}} u_{j} u_{k} d x-0,
$$

whenever $j \neq k$, using here that $u_{j}$ and $u_{k}$ are orthogonal and $T^{-1}$ has constant Jacobian. Thus by the Rayleigh-Poincaré principle, we have

$$
\sum_{j=1}^{n} \lambda_{j}(\Omega) \leq \sum_{j=1}^{n} \frac{\int_{\Omega}\left|\nabla v_{j}\right|^{2} d x}{\int_{\Omega} v_{j}^{2} d x}
$$

The denominator of this Rayleigh quotient is

$$
\int_{\Omega} v_{j}^{2} d x=\int_{\Omega} u_{j}\left(U^{-1} T(x)\right)^{2} d x=\operatorname{Jac}\left(T^{-1}\right)
$$

by (17) with $j=k$, since the eigenfunctions are normalized with

$$
\int_{\mathbb{B}} u_{j}^{2} d x=1
$$


For the numerator of the Rayleigh quotient, we write $v=v_{j}$ and $u=u_{j}$ (to simplify notation in what follows) and express $v=v(r, \xi)$ and $u=u(s, \zeta)$ in spherical coordinates. Then the relation $v=u \circ U^{-1} \circ T$ says

$$
v(r, \xi)=u\left(r \Gamma(\xi), U^{-1} H(\xi)\right),
$$

by recalling that $\Gamma=1 / R$ and using the definition (5) of $T$. Differentiating, we find

$$
v_{r}(r, \xi)=\Gamma(\xi) u_{s}\left(r \Gamma(\xi), U^{-1} H(\xi)\right),
$$

and

$$
\begin{aligned}
\left(\nabla_{\S} v\right)(r, \xi)= & r u_{s}\left(r \Gamma(\xi), U^{-1} H(\xi)\right) \nabla \Gamma(\xi) \\
& +\left(\nabla_{\S} u\right)\left(r \Gamma(\xi), U^{-1} H(\xi)\right) U^{-1} D H(\xi),
\end{aligned}
$$

where the gradients are regarded as row vectors and by $\nabla_{\S}$ we mean the gradient with respect to the angular variables. Writing the numerator of the Rayleigh quotient in terms of spherical coordinates gives that

$$
\int_{\Omega}|\nabla v|^{2} d x=\int_{\Phi} \int_{0}^{R(\xi)}\left(v_{r}^{2}+r^{-2}\left|\nabla_{\S} v\right|^{2}\right) r^{d-1} d r d S(\xi) .
$$

After changing variable with $s=r \Gamma(\xi) \in(0,1)$ and using the above formulas for $v_{r}$ and $\nabla_{\S} v$, we find that

$$
\int_{\Omega}|\nabla v|^{2} d x=Q_{1}+Q_{2}+Q_{3}
$$

where

$$
\begin{aligned}
& Q_{1}=\int_{\Phi} \int_{0}^{1}\left[\Gamma(\xi)^{2}+|\nabla \Gamma(\xi)|^{2}\right] u_{s}\left(s, U^{-1} H(\xi)\right)^{2} s^{d-1} d s R(\xi)^{d} d S(\xi), \\
& Q_{2}=2 \int_{\Phi} \int_{0}^{1} \Gamma(\xi) \nabla \Gamma(\xi)(D H)^{\dagger}(\xi) U \\
& Q_{3}=\int_{\Phi} \int_{0}^{1} \Gamma\left(\nabla_{\S} u\right)^{\dagger}\left(s, U^{-1} H(\xi)\right) u_{s}\left(s, U^{-1} H(\xi)\right) s^{d-2} d s R(\xi)^{d} d S(\xi),
\end{aligned}
$$

The left side of (18) is independent of $U$. Hence by averaging (18) with respect to $U \in O(d)$ we find

$$
\sum_{j=1}^{n} \lambda_{j}(\Omega) \leq \sum_{j=1}^{n} \frac{\int_{O(d)}\left(Q_{1}+Q_{2}+Q_{3}\right) d \gamma(U)}{\operatorname{Jac}\left(T^{-1}\right)},
$$

where we must remember that " $u$ " means $u_{j}$, in the quantities $Q_{1}, Q_{2}, Q_{3}$. 
The quantity $Q_{1}$ is easiest to average because it contains only one " $U$ ". We have

$$
\int_{O(d)} u_{s}\left(s, U^{-1} H(\xi)\right)^{2} d \gamma(U)=\frac{1}{|\mathbb{\$}|} \int_{\Phi} u_{S}(s, \zeta)^{2} d S(\zeta)
$$

by the equivalence of orbital and spatial means (Appendix B). Hence

$$
\begin{aligned}
& \frac{\int_{O(d)} Q_{1} d \gamma(U)}{\operatorname{Jac}\left(T^{-1}\right)} \\
& =\frac{\frac{1}{|\mathbb{S}|} \int_{\mathbb{S}}\left[\Gamma(\xi)^{2}+|\nabla \Gamma(\xi)|^{2}\right] R(\xi)^{d} d S(\xi)}{V(\Omega) / V(\mathbb{B})} \int_{0}^{1} \int_{\Phi} u_{s}(s, \zeta)^{2} d S(\zeta) s^{d-1} d s \\
& =G_{0}(\Omega)\left(\frac{V(\mathbb{B})}{V(\Omega)}\right)^{2 / d} \int_{\mathbb{B}}\left(\frac{\partial u_{j}}{\partial s}\right)^{2} d x
\end{aligned}
$$

by the definition (6) of $G_{0}$.

For $Q_{2}$ we begin by changing variable with $\xi=H^{-1}(U \zeta)$, which gives that

$$
\begin{gathered}
Q_{2}=\int_{\Phi} \int_{0}^{1} \Gamma\left(H^{-1}(U \zeta)\right) \nabla \Gamma\left(H^{-1}(U \zeta)\right)(D H)^{\dagger}\left(H^{-1}(U \zeta)\right) U \\
\left(\nabla_{\Phi} u\right)^{\dagger}(s, \zeta) u_{s}(s, \zeta) s^{d-2} d s \frac{R\left(H^{-1}(U \zeta)\right)^{d}}{\operatorname{Jac}_{H}\left(H^{-1}(U \zeta)\right)} d S(\zeta) .
\end{gathered}
$$

The integrand contains $U$ in multiple locations, but averaging remains feasible; indeed Proposition 5.2 applied with $F=(\Gamma \nabla \Gamma) R^{d} / \mathrm{Jac}_{H}$ shows that

$$
\int_{O(d)} Q_{2} d \gamma(U)=0
$$

For $Q_{3}$ we again change variable with $\xi=H^{-1}(U \zeta)$, and find that

$$
\begin{gathered}
Q_{3}=\int_{\Phi} \int_{0}^{1} \Gamma\left(H^{-1}(U \zeta)\right)^{2}\left|\nabla_{\S} u(s, \zeta) U^{-1} D H\left(H^{-1}(U \zeta)\right)\right|^{2} s^{d-3} d s \\
\frac{R\left(H^{-1}(U \zeta)\right)^{d}}{\operatorname{Jac}_{H}\left(H^{-1}(U \zeta)\right)} d S(\zeta) .
\end{gathered}
$$

In this integrand $U$ appears five times. Nonetheless, we can average $Q_{3}$ with respect to $U$ by expanding $|\cdots|^{2}$ and using Proposition 5.2 with $f=\Gamma^{2} R^{d} / \mathrm{Jac}_{H}$. We find that

$$
\int_{O(d)} Q_{3} d \gamma(U)=c \int_{\Phi} \int_{0}^{1}\left(\nabla_{\Phi} u\right)\left(\mathrm{Id}-\zeta \zeta^{\dagger}\right)\left(\nabla_{\Phi} u\right)^{\dagger} s^{d-3} d s d S(\zeta)
$$


where

$$
c=\frac{1}{|\Phi|} \int_{\Phi} \Gamma(\xi)^{2} \frac{\|D H(\xi)\|_{H S}^{2}}{d-1} R(\xi)^{d} d S(\xi) .
$$

[Aside. The averaging results (21) and (22) for $Q_{2}$ and $Q_{3}$ hold independently of the specific form of the Jacobian of $H$, provided the Jacobian is bounded away from zero (since we want to avoid trouble when we divide by it).]

Note that $\left(\nabla_{\S} u\right) \zeta=0$, because the spherical gradient $\nabla_{\S} u$ lies perpendicular to the unit vector $\zeta$. Hence we deduce that

$$
\begin{aligned}
\frac{\int_{O(d)} Q_{3} d \gamma(U)}{\operatorname{Jac}\left(T^{-1}\right)} & =\frac{V(\mathbb{B})}{V(\Omega)} c \int_{\Phi} \int_{0}^{1}\left(\nabla_{\S} u\right)\left(\nabla_{\S} u\right)^{\dagger} s^{d-3} d s d S(\zeta) \\
& =G_{1}(\Omega)\left(\frac{V(\mathbb{B})}{V(\Omega)}\right)^{2 / d} \int_{\mathbb{B}} s^{-2}\left|\nabla_{\S} u_{j}\right|^{2} d x
\end{aligned}
$$

by definition of $G_{1}$ in (7).

Combining (19)-(23) now shows that

$$
\begin{aligned}
\sum_{j=1}^{n} \lambda_{j}(\Omega) & \leq\left(\frac{V(\mathbb{B})}{V(\Omega)}\right)^{2 / d} \sum_{j=1}^{n}\left[G_{0}(\Omega) \int_{\mathbb{B}}\left(\frac{\partial u_{j}}{\partial s}\right)^{2} d x+G_{1}(\Omega) \int_{\mathbb{B}} s^{-2}\left|\nabla_{\S} u_{j}\right|^{2} d x\right] \\
& =\left(\frac{V(\mathbb{B})}{V(\Omega)}\right)^{2 / d} \sum_{j=1}^{n}\left[\left(1-\alpha_{j}\right) G_{0}(\Omega)+\alpha_{j} G_{1}(\Omega)\right] \int_{\mathbb{B}}\left|\nabla u_{j}\right|^{2} d x,
\end{aligned}
$$

where

$$
\alpha_{j}=\frac{\int_{\mathbb{B}} s^{-2}\left|\nabla_{\Phi} u_{j}\right|^{2} d x}{\int_{\mathbb{B}}\left|\nabla u_{j}\right|^{2} d x}, \quad j=1,2, \ldots, n .
$$

(The coefficient $\alpha_{j} \in[0,1]$ measures the "angular component" of the $j$ th energy.) Next we estimate $G_{0}$ and $G_{1}$ from above with their maximum $G$, and so conclude that

$$
\sum_{j=1}^{n} \lambda_{j}(\Omega) V(\Omega)^{2 / d} / G(\Omega) \leq V(\mathbb{B})^{2 / d} \sum_{j=1}^{n} \int_{\mathbb{B}}\left|\nabla u_{j}\right|^{2} d x=\sum_{j=1}^{n} \lambda_{j}(\mathbb{B}) V(\mathbb{B})^{2 / d} .
$$

Since $G(\mathbb{B})=1$, we have proved the theorem in the case that $\Phi(a) \equiv a$ is the identity function.

The theorem now follows for any concave increasing $\Phi$, by Hardy-LittlewoodPólya majorization as in Appendix A.

Note that for the first eigenvalue our proof gives a stronger conclusion, namely using $G_{0}$ instead of $G$, because the fundamental mode $u_{1}$ of the ball is a radial function and so $\alpha_{1}=0$ in the argument above. Thus $\lambda_{1} V^{2 / d} / G_{0}$ is maximal for the ball. 
For the second Dirichlet eigenvalue, we may divide and multiply by the first eigenvalue to obtain that

$$
\frac{\lambda_{2} V^{2 / d}}{G_{0}}=\frac{\lambda_{1} V^{2 / d}}{G_{0}} \frac{\lambda_{2}}{\lambda_{1}} .
$$

We previously showed that the first factor is maximal for the ball, and the second factor is maximal too, by the sharp Payne-Pólya-Weinberger result of Ashbaugh and Benguria [2].

Particular cases. Applying the theorem with $\Phi(a)=a^{s}$, which is concave and increasing when $0<s \leq 1$, gives maximality of $\left(\lambda_{1}^{s}+\cdots+\lambda_{n}^{s}\right)^{1 / s} V^{2 / d} / G$ for the ball. The limiting case $s \downarrow 0$ suggests we try choosing $\Phi(a)=\log a$, which yields maximality of the ball for the functional

$$
\sum_{j=1}^{n} \log \left(\lambda_{j} V^{2 / d} / G\right)=n \log \left(\sqrt[n]{\lambda_{1} \cdots \lambda_{n}} V^{2 / d} / G\right) .
$$

When $s<0$ we can choose the concave increasing function $\Phi(a)=-a^{s}$, which leads to minimality of the ball for $\sum_{j=1}^{n}\left(\lambda_{j} V^{2 / d} / G\right)^{s}$. And for $t>0$ we can consider $\Phi(a)=-e^{-a t}$, thus obtaining minimality at the ball of $\sum_{j=1}^{n} \exp \left(-\lambda_{j} V^{2 / d} t / G\right)$.

Dirichlet equality statement. Assume equality holds for the first eigenvalue, that is,

$$
\lambda_{1} V^{2 / d} /\left.G_{0}\right|_{\Omega}=\lambda_{1} V^{2 / d} /\left.G_{0}\right|_{\mathbb{B}} .
$$

By enforcing equality in our proof above, we see that the trial function $v_{1}$ on $\Omega$ must attain equality in the Rayleigh characterization of $\lambda_{1}(\Omega)$, and hence must be a first eigenfunction for $\Omega$. In particular this holds when the orthogonal matrix $U$ is the identity, so that the function $v_{1}(x)=u_{1}(T(x))$ satisfies

$$
\Delta v_{1}=-\lambda_{1}(\Omega) v_{1} .
$$

The fundamental Dirichlet mode $u_{1}$ of the ball is radial, with $u_{1}(x)=J(|x|)$ for some positive function $J$, and so we have $v_{1}(x)=J(r / R(\xi))$. That is,

$$
v_{1}(x)=J(r \Gamma(\xi))
$$

where $\Gamma=1 / R$. Note $R$ is $C^{2}$-smooth by assumption, in this part of the theorem.

The Laplacian of $v_{1}$ is given in spherical coordinates by

$$
\Delta v_{1}(x)=J^{\prime \prime}(r \Gamma(\xi)) \Gamma(\xi)^{2}+\frac{d-1}{r} J^{\prime}(r \Gamma(\xi)) \Gamma(\xi)+\frac{1}{r^{2}} \Delta_{\Phi}[J(r \Gamma(\xi))] .
$$

This spherical Laplacian can be computed by the chain rule. It equals

$$
\Delta_{\Phi}[J(r \Gamma(\xi))]=J^{\prime \prime}(r \Gamma(\xi)) r^{2}|\nabla \Gamma(\xi)|^{2}+J^{\prime}(r \Gamma(\xi)) r \Delta_{\Phi} \Gamma(\xi) .
$$


We substitute this formula into the preceding one, and make the substitution $s=$ $r \Gamma(\xi)$. Then the eigenfunction equation (25) reads

$$
\left[\Gamma(\xi)^{2}+|\nabla \Gamma(\xi)|^{2}\right] J^{\prime \prime}(s)+\left[(d-1) \Gamma(\xi)^{2}+\Gamma(\xi) \Delta_{\S} \Gamma(\xi)\right] \frac{1}{s} J^{\prime}(s)=-\lambda_{1}(\Omega) J(s) .
$$

Integrating over $\xi \in \mathbb{\$}$ yields that

$$
\|\Gamma\|_{H^{1}}^{2} J^{\prime \prime}(s)+\left[(d-1)\|\Gamma\|_{2}^{2}-\|\nabla \Gamma\|_{2}^{2}\right] \frac{1}{s} J^{\prime}(s)=-\lambda_{1}(\Omega)|\$| J(s) .
$$

The eigenfunction equation for the unit ball (the case $\Gamma \equiv 1$ ) says that

$$
J^{\prime \prime}(s)+\frac{d-1}{s} J^{\prime}(s)=-\lambda_{1}(\mathbb{B}) J(s) .
$$

We subtract $\|\Gamma\|_{H^{1}}^{2}$ times this equation from the previous equation, thereby obtaining a first order equation for $J$ :

$$
\|\nabla \Gamma\|_{2}^{2} J^{\prime}(s)=\frac{1}{d}\left(\lambda_{1}(\Omega)|\$|-\lambda_{1}(\mathbb{B})\|\Gamma\|_{H^{1}}^{2}\right) s J(s), \quad 0<s<1 .
$$

Suppose $\Gamma \not \equiv$ const., which ensures that $\|\nabla \Gamma\|_{2}>0$. Then the last equation for $J$ has the form

$$
J^{\prime}(s)=a s J(s)
$$

for some $a \in \mathbb{R}$, and so $J^{\prime \prime}(s)=\left(a^{2} s^{2}+a\right) J(s)$. Substituting these relations into the eigenfunction equation (26) implies $a^{2} s^{2}+a d=-\lambda_{1}(\mathbb{B})$ for all $s \in(0,1)$, and so $a=0$ and then $\lambda_{1}(\mathbb{B})=0$. This contradiction tells us that $\Gamma \equiv$ const., and so $R \equiv$ const., which means that $\Omega$ is a centered ball.

\section{Neumann eigenvalues - Proof of Theorem 3.3}

For Neumann boundary conditions, the Rayleigh quotient and Rayleigh-Poincaré Principle are just as for the Dirichlet case, except using trial functions in $H^{1}(\Omega)$ rather than $H_{0}^{1}(\Omega)$. Thus we may follow the proof of Theorem 3.1, except using Neumann eigenfunctions of the ball instead of Dirichlet eigenfunctions, to prove that

$$
\sum_{j=1}^{n} \mu_{j}(\Omega) V(\Omega)^{2 / d} / G(\Omega) \leq \sum_{j=1}^{n} \mu_{j}(\mathbb{B}) V(\mathbb{B})^{2 / d} .
$$

On each side, the term with $j=1$ may now be discarded because $\mu_{1}=0$. Then the proof can be completed by majorization.

For the equality statement, rather than adapting the Dirichlet case we present a simpler approach. Suppose equality holds for the first nonzero eigenvalue, that is,

$$
\mu_{2} V^{2 / d} /\left.G\right|_{\Omega}=\mu_{2} V^{2 / d} /\left.G\right|_{\mathbb{B}} .
$$


Since $\left.\mu_{2} V^{2 / d}\right|_{\Omega} \leq\left.\mu_{2} V^{2 / d}\right|_{\mathbb{B}}$ by the Szegó-Weinberger result [41] (or see [19], Theorem 7.1.1), and since $G(\Omega) \geq 1=G(\mathbb{B})$, we conclude from equality holding in (27) that $G(\Omega)=1$. Hence $G_{0}(\Omega)=1$, and so $\Omega$ is a centered ball by the equality statement in Lemma 2.2.

\section{Robin eigenvalues - Proof of Theorem 3.5}

The Rayleigh quotient for the Robin problem is

$$
\operatorname{Ray}[u]=\frac{\hbar^{2} \int_{\Omega}|\nabla u|^{2} d x+\int_{\partial \Omega} \sigma u^{2} d S}{\int_{\Omega} u^{2} d x}, \quad u \in H^{1}(\Omega) .
$$

Let $\left\{u_{j}\right\}$ be orthonormal eigenfunctions on the unit ball $\mathbb{B}$ that correspond to the Robin eigenvalues $\rho_{j}\left(\mathbb{B}, \hbar V(\mathbb{B})^{1 / d}, \bar{\sigma} V(\mathbb{B})^{1 / d}\right)$, for $j=1,2,3, \ldots$ By constructing trial functions and using the Rayleigh-Poincaré principle as in the Dirichlet case (Section 6), we find the following analogue of (18):

$$
\begin{aligned}
& \sum_{j=1}^{n} \rho_{j}\left(\Omega, \hbar V^{1 / d} / G^{1 / 2}, \sigma V^{1 / d} / G_{\text {Robin }}^{1 / 2}\right) \\
& \leq \sum_{j=1}^{n} \frac{\hbar^{2} V^{2 / d}}{G} \frac{\int_{\Omega}\left|\nabla v_{j}\right|^{2} d x}{\int_{\Omega} v_{j}^{2} d x}+\sum_{j=1}^{n} \frac{V^{1 / d}}{G_{\text {Robin }}^{1 / 2}} \frac{\int_{\partial \Omega} \sigma v_{j}^{2} d S}{\int_{\Omega} v_{j}^{2} d x},
\end{aligned}
$$

where $v_{j}=u_{j} \circ U^{-1} \circ T$. Averaging over $U \in O(d)$ (as explained in Section 6 leading up to (24)) shows that the first sum in (29) is bounded from above by

$$
\sum_{j=1}^{n} \hbar^{2} V(\mathbb{B})^{2 / d} \int_{\mathbb{B}}\left|\nabla u_{j}\right|^{2} d x .
$$

For the principal eigenvalue $(n=1)$ this part of the argument also works with $G_{0}$ in place of $G$, since $u_{1}$ is radial. (When finding $u_{1}$ by separation of variables, the spherical harmonics with angular dependence cannot arise, because $u_{1}$ is positive. Hence $u_{1}$ is radial.)

We will show below that averaging the second sum in (29) gives

$$
\sum_{j=1}^{n} \bar{\sigma} V(\mathbb{B})^{1 / d} \int_{\Phi} u_{j}^{2} d S .
$$


The theorem then follows, because adding (30) and (31) gives

$$
\sum_{j=1}^{n} \rho_{j}\left(\mathbb{B}, \hbar V(\mathbb{B})^{1 / d}, \bar{\sigma} V(\mathbb{B})^{1 / d}\right) .
$$

For (31) it suffices to consider one value of $j$ at a time, and so we consider an arbitrary function $u \in H^{1}(\Omega)$ with $L^{2}$-norm equal to 1 , and write $v=u \circ U^{-1} \circ T$. The second sum in (29) has terms of the form

$$
\frac{V^{1 / d}}{G_{\text {Robin }}^{1 / 2}} \frac{\int_{\partial \Omega} \sigma v^{2} d S}{\int_{\Omega} v^{2} d x}=\frac{V^{1 / d}}{G_{\text {Robin }}^{1 / 2}} \frac{\int_{\partial \Omega} \sigma u\left(U^{-1} T(x)\right)^{2} d S(x)}{V(\Omega) / V(\mathbb{B})},
$$

where in the denominator we changed variable and used that $T$ has constant Jacobian and $u$ has $L^{2}$-norm equal to 1 . Averaging the right side of (32) over matrices $U \in$ $O(d)$ gives (by the equivalence of orbital and spatial means, as in Appendix B) the expression

$$
\frac{V(\Omega)^{1 / d}}{G_{\text {Robin }}(\Omega)^{1 / 2}} \frac{V(\mathbb{B})}{V(\Omega)}\left(\int_{\partial \Omega} \sigma d S(x)\right)\left(\frac{1}{|\mathbb{S}|} \int_{\mathbb{S}} u^{2} d S\right) .
$$

This last expression equals $V(\mathbb{B})^{1 / d} \bar{\sigma} \int_{\Phi} u^{2} d S$ by definition of $G_{\text {Robin }}$ and $\bar{\sigma}$, proving (31).

To prove inequality (9) for the first eigenvalue, apply the theorem with $n=1$ and $G_{0}$ instead of $G$ (as remarked above), and replace $\hbar$ by $\hbar / V(\mathbb{B})^{1 / d}$ and replace $\sigma$ by $\sigma / V(\mathbb{B})^{1 / d}$.

For the equality statement on the first eigenvalue, one simply adapts the proof of the Dirichlet equality statement in Section 6.

\section{Improvement to the main results}

Our main theorems attach the geometric factor $G=\max \left\{G_{0}, G_{1}\right\}$ to each eigenvalue. An inspection of the proofs yields a stronger result, in which each eigenvalue is paired with a smaller geometric factor arising from a convex combination of $G_{0}$ and $G_{1}$. We state this improved result below, restricting for simplicity to the case of eigenvalue sums. (The reader can then deduce inequalities on spectral zeta functions and so on, by applying the majorization result from Appendix A.) To simplify the exposition we do not treat the Robin case.

Define a convex combination of the geometric factors by

$$
G(\alpha ; \Omega)=(1-\alpha) G_{0}(\Omega)+\alpha G_{1}(\Omega), \quad \alpha \in[0,1] .
$$


To choose the relevant values of $\alpha$, we fix an orthonormal basis of eigenfunctions $u_{1}, u_{2}, u_{3}, \ldots$ of the unit ball $\mathbb{B}$ corresponding to the Dirichlet eigenvalues $\lambda_{1}(\mathbb{B}), \lambda_{2}(\mathbb{B}), \lambda_{3}(\mathbb{B}), \cdots$. The radial energy fraction of the $j$ th Dirichlet eigenfunction is defined to be

$$
\varepsilon_{j}^{D}=\frac{\int_{\mathbb{B}}\left(\partial u_{j} / \partial s\right)^{2} d x}{\int_{\mathbb{B}}\left|\nabla u_{j}\right|^{2} d x},
$$

where $s \in[0,1]$ denotes the radial variable. This energy fraction can be computed explicitly by writing $u_{j}$ in terms of Bessel functions. Obviously $0<\varepsilon_{j}^{D} \leq 1$, with $\varepsilon_{j}^{D}=1$ if and only if $u_{j}$ is purely radial.

The angular energy fraction is then

$$
\alpha_{j}^{D}=1-\varepsilon_{j}^{D}
$$

For example, the principal Dirichlet mode of the ball is radial, and so $\alpha_{1}^{D}=0$.

Similarly, we may define the angular energy fraction $\alpha_{j}^{N}$ for the Neumann eigenfunctions of the unit ball.

Theorem 9.1 (Improved inequalities). Assume $\Omega$ is a Lipschitz-starlike domain in $\mathbb{R}^{d}$. Then the Dirichlet and Neumann eigenvalues satisfy

$$
\sum_{j=1}^{n} \lambda_{j}(\Omega) V(\Omega)^{2 / d} \leq \sum_{j=1}^{n} \lambda_{j}(\mathbb{B}) V(\mathbb{B})^{2 / d} G\left(\alpha_{j}^{D} ; \Omega\right), \quad n \geq 1
$$

and

$$
\sum_{j=2}^{n} \mu_{j}(\Omega) V(\Omega)^{2 / d} \leq \sum_{j=2}^{n} \mu_{j}(\mathbb{B}) V(\mathbb{B})^{2 / d} G\left(\alpha_{j}^{N} ; \Omega\right), \quad n \geq 2
$$

Proof of Theorem 9.1. See (24) in the proof of Theorem 3.1. The Neumann case is analogous.

\section{Properties of the geometric factors}

$\boldsymbol{G}_{1}$ does not depend on $\boldsymbol{H}$ in $\mathbf{2}$ dimensions. The quantity $G_{1}$ defined in (7) depends only on $R$ and not on $H$, in 2 dimensions, by the following result.

Proposition 10.1. In dimension $d=2$,

$$
G_{0}=1+\frac{1}{2 \pi} \int_{0}^{2 \pi}(\log R)^{\prime}(\theta)^{2} d \theta
$$


and

$$
G_{1}=\frac{\frac{1}{2 \pi} \int_{0}^{2 \pi} R(\theta)^{4} d \theta}{\left(\frac{1}{2 \pi} \int_{0}^{2 \pi} R(\theta)^{2} d \theta\right)^{2}}=\frac{2 \pi I_{\text {origin }}}{A^{2}},
$$

where $A$ is the area of $\Omega$ and

$$
I_{\text {origin }}=\int_{\Omega}|x|^{2} d A
$$

is its polar moment of inertia about the origin. Further,

$$
G_{\text {Robin }}=\frac{L^{2}}{4 \pi A}
$$

where $L$ is the perimeter of $\Omega$.

These formulas imply immediately that $G_{0}, G_{1}, G_{\text {Robin }}$ are $\geq 1$ and are scale invariant with respect to dilations of the domain, in 2 dimensions.

Proof of Proposition 10.1. To prove the first and third formulas, simply substitute $d=2$ into the definitions (6) and (8) of $G_{0}$ and $G_{\text {Robin }}$.

For the second formula, when $d=2$ the definition (7) of $G_{1}$ implies

$$
G_{1}=\int_{0}^{2 \pi}\left\|D H\left(\begin{array}{c}
\cos \theta \\
\sin \theta
\end{array}\right)\right\|_{H S}^{2} d \theta / 2 \pi .
$$

The homeomorphism $H$ of the unit circle can be written $H\left(\begin{array}{c}\cos \theta \\ \sin \theta\end{array}\right)=\left(\begin{array}{c}\cos \varphi(\theta) \\ \sin \varphi(\theta)\end{array}\right)$. Homogeneity of $H$ then gives $H\left(\begin{array}{c}x_{1} \\ x_{2}\end{array}\right)=\left(\begin{array}{c}\cos \varphi(\theta) \\ \sin \varphi(\theta)\end{array}\right)$ where $\theta=\arg \left(x_{1}+i x_{2}\right)=$ $\arctan \left(x_{2} / x_{1}\right)$. Calculating the derivative matrix $D H\left(\begin{array}{l}x_{1} \\ x_{2}\end{array}\right)$ results in

$$
\left\|D H\left(\begin{array}{c}
\cos \theta \\
\sin \theta
\end{array}\right)\right\|_{H S}^{2}=\varphi^{\prime}(\theta)^{2} .
$$

Further, the distortion formula (4) for $H$ says in 2 dimensions that

$$
\varphi^{\prime}(\theta)^{2}=\left(\frac{A(\mathbb{B})}{A(\Omega)} R(\theta)^{2}\right)^{2} .
$$

Substituting the last two formulas into (35) shows that

$$
G_{1}=\frac{\int_{0}^{2 \pi} R(\theta)^{4} d \theta / 2 \pi}{(A(\Omega) / \pi)^{2}} .
$$

Now (34) follows by evaluating area and moment of inertia in polar coordinates. 
Expressing $G_{0}$ as a support-type functional, in all dimensions. The geometric meaning of $G_{0}$ is highlighted by the following lemma.

Lemma 10.2 (Equivalence of our $G_{0}$ with the definitions of Pólya-Szegó and Freitas-Krejčiř́k). If $\Omega$ is a Lipschitz-starlike domain then

$$
G_{0}=\frac{1}{|\mathbb{S}|} \int_{\partial \Omega} \frac{1}{x \cdot N(x)} d S(x)\left(\frac{V(\mathbb{B})}{V(\Omega)}\right)^{(d-2) / d} .
$$

Thus in 2 dimensions,

$$
G_{0}=\frac{1}{2 \pi} \int_{\partial \Omega} \frac{1}{x \cdot N(x)} d s(x) .
$$

Hence if $\Omega$ is convex then $G_{0} \leq\left(V / V_{i n}\right)^{2 / d}$, where $V_{i n}$ is the volume of the largest open ball centered at the origin and contained in $\Omega$. .

Pólya and Szegő's calculations already prove the lemma in dimension 2 (see [38], p. 92), but the lemma is new in higher dimensions because Freitas and Krejčiřík proceeded along somewhat different lines in their proof.

Proof of Lemma 10.2. Our first task is to evaluate $x \cdot N(x)$ in terms of the radius function. The boundary of $\Omega$ is the level set $\left\{x:|x|^{2}=R(x)^{2}\right\}$ and so taking the gradient gives an outward normal vector $n(x)=x-R(x) \nabla R(x)$. We evaluate at $x=R(\xi) \xi \in \partial \Omega$ to obtain $n(x)=R(\xi) \xi-R(\xi) \nabla R(R(\xi) \xi)$. The homogeneity relation $R(r \xi)=R(\xi)$ implies that $r \nabla R(r \xi)=\nabla R(\xi)$ for each $r>0$, and so $n(x)=R(\xi) \xi-\nabla R(\xi)$. Thus for the unit normal $N(x)=n(x) /|n(x)|$ we compute

$$
x \cdot N(x)=\frac{R(\xi)^{2}}{\sqrt{R(\xi)^{2}+|\nabla R(\xi)|^{2}}} .
$$

where we used that $\xi \cdot \nabla R(\xi)=0$ (by homogeneity of $R$ ).

Next we need a formula for surface area element on the boundary of $\Omega$ :

$$
d S(x)=R(\xi)^{d-2} \sqrt{R(\xi)^{2}+|\nabla R(\xi)|^{2}} d S(\xi),
$$

as one proves straightforwardly by parameterizing $\partial \Omega$ as $\{x=R(\xi) \xi: \xi \in \mathbb{\$}\}$.

By substituting the preceding formulas into the formula for $G_{0}$ in the lemma, we see that it reduces to the definition of $G_{0}$ in Section 2.

Now write $\mathbb{B}_{i n}$ for the ball of volume $V_{i n}$ centered at the origin, and write $R_{i n}$ for its radius. If $\Omega$ is convex then $R_{i n} \leq x \cdot N(x)$ for all $x \in \Omega$, as one sees by considering a support plane at $x$, and so

$$
\frac{1}{x \cdot N(x)} \leq \frac{x \cdot N(x)}{R_{i n}^{2}}
$$

By integrating over $\partial \Omega$ and using the formula in the lemma for $G_{0}$, on the left side, and the divergence theorem on the right side, we find that $G_{0} \leq\left(V / V_{i n}\right)^{2 / d}$. 
Evaluation of $\boldsymbol{G}_{\mathbf{0}}$ for polygons with an inscribed circle. If $\Omega$ is a triangle with incenter at the origin, or more generally if $\Omega$ is any polygon with an inscribed circle centered at the origin, then the geometric factor $G_{0}$ can be evaluated in terms of area and perimeter. For such domains $G_{0}=L^{2} / 4 \pi A=G_{\text {Robin }}$, as was proved by Aissen [1], Theorem 1. To prove this fact observe that $x \cdot N(x)$ equals the inradius, for each point $x$ on the boundary. Hence formula (36) gives that $G_{0}$ equals $L / 2 \pi$ divided by the inradius, which evaluates to $L^{2} / 4 \pi A$ because $A=\frac{1}{2} L R_{i n}$ (by triangulating the domain with respect to the origin).

Proof that $G_{0} \geq G_{\text {Robin }} \geq 1$ and $G_{1} \geq 1$ (for Lemmas 2.2 and 3.4). We have from Lemma 10.2 and Cauchy-Schwarz that

$$
\begin{aligned}
G_{0} & \geq \frac{1}{|\mathbb{S}|} \frac{|\partial \Omega|^{2}}{\int_{\partial \Omega} x \cdot N(x) d S(x)}\left(\frac{V(\mathbb{B})}{V(\Omega)}\right)^{(d-2) / d} \\
& =\frac{1}{|\$|} \frac{|\partial \Omega|^{2}}{V(\Omega) d}\left(\frac{V(\mathbb{B})}{V(\Omega)}\right)^{(d-2) / d} \\
& =G_{\text {Robin }}
\end{aligned}
$$

by the divergence theorem (noting $\nabla \cdot x \equiv d$ ), and by definition of $G_{\text {Robin }}$ in (8). Note that if equality holds then $x \cdot N(x)$ is constant, by the equality conditions for Cauchy-Schwarz.

Further, $G_{\text {Robin }} \geq 1$ by the isoperimetric inequality, as remarked after (8), with equality if and only if $\Omega$ is a ball.

Hence $G_{0} \geq G_{\text {Robin }} \geq 1$, and $G_{0}=1$ if and only if $\Omega$ is a centered ball.

(Aside. This inequality was established in 2 dimensions by Aissen [1], Theorem 1.)

Now we prove $G_{1} \geq 1$. By applying the quadratic-geometric mean inequality to the nonzero singular values of $D H$ we deduce that

$$
\frac{\|D H(\xi)\|_{H S}^{2}}{d-1} \geq \operatorname{Jac}_{H}(\xi)^{2 /(d-1)}=\left(\frac{V(\mathbb{B})}{V(\Omega)}\right)^{2 /(d-1)} R(\xi)^{2 d /(d-1)},
$$

where the last step uses the distortion formula for $H$ in (4). Substituting this estimate into the definition of $G_{1}$ in (7) shows that

$$
\begin{aligned}
G_{1} & \geq\left(\frac{V(\mathbb{B})}{V(\Omega)}\right)^{2 /(d-1)}\left(\frac{1}{|\mathbb{}|} \int_{\mathbb{S}}\left(R^{d}\right)^{1+2 / d(d-1)} d S\right) /\left(\frac{1}{|\mathbb{S}|} \int_{\mathbb{S}} R^{d} d S\right)^{(d-2) / d} \\
& \geq\left(\frac{V(\mathbb{B})}{V(\Omega)}\right)^{2 /(d-1)}\left(\frac{1}{|\mathbb{\$}|} \int_{\mathbb{S}} R^{d} d S\right)^{2 /(d-1)}
\end{aligned}
$$

by Jensen's inequality. Since $\int_{\mathbb{S}} R^{d} d S=V(\Omega) d$ and $|\$|=V(\mathbb{B}) d$, we deduce that $G_{1} \geq 1$. 
If $G_{1}=1$ then $R$ is constant (by the equality conditions for Jensen), and so $\Omega$ is a centered ball. Then $H$ maps $\mathbb{S}$ to $\$$ with constant distortion $\mathrm{Jac}_{H} \equiv 1$, by (4). In 2 dimensions that is enough to imply $H$ is an orthogonal transformation (cf. Section 11). So suppose $d \geq 3$. Note the nonzero singular values of $D H$ all have equal magnitude (and hence have magnitude 1), by the equality conditions for the quadratic-geometric mean inequality. Therefore by the singular value decomposition, $\mathrm{DH}$ acts as an orthogonal matrix on the tangent space, at almost every point of the sphere. Liouville's theorem [21] implies that $H$ is a Möbius transformation that fixes the sphere. Since also the Jacobian of $H$ equals 1 identically on the sphere, we conclude that $H$ is an orthogonal transformation.

Good choices of origin. What is a good choice of origin within the domain, given that for Theorem 3.1 we would like to make the geometric factors $G_{0}$ and $G_{1}$ as small as possible?

To minimize $G_{1}$ one should choose the origin at the center of mass, because Proposition 10.1 expresses $G_{1}$ in terms of moment of inertia (at least in 2 dimensions).

The center of mass is not generally the best choice of origin for $G_{0}$. For a polygon with an inscribed circle, $G_{0}$ is minimal when the origin coincides with the center of the circle; this observation is due to Aissen [1], §3, with the key step being the use of Cauchy-Schwarz as in (37). For a triangle, for example, the inscribed circle is centered where the angle bisectors intersect, which can be quite far from the center of mass (as happens for a thin acute isosceles triangle). Thus in general one cannot hope to minimize both $G_{0}$ and $G_{1}$ with a single choice of origin. An exception is for domains having two axes of symmetry, in which case both factors are minimized when the origin is at the intersection (for $G_{1}$ because the intersection point is the centroid, and for $G_{0}$ by work of Aissen [1], Corollaries 1, 2, and 3).

The question of whether $G_{0}$ or $G_{1}$ is larger can be subtle to resolve. For example, consider the ellipse with semi-axes 3 and 1, shown in Figure 3. Choosing the origin at the center would minimize both factors, and in fact would make them equal (as one finds by direct computation). Nearby choices of origin, though, could lead to either $G_{0}$ or $G_{1}$ being larger. Thus for domains like perturbed ellipses it is unclear which factor will dominate, until computations have been performed.

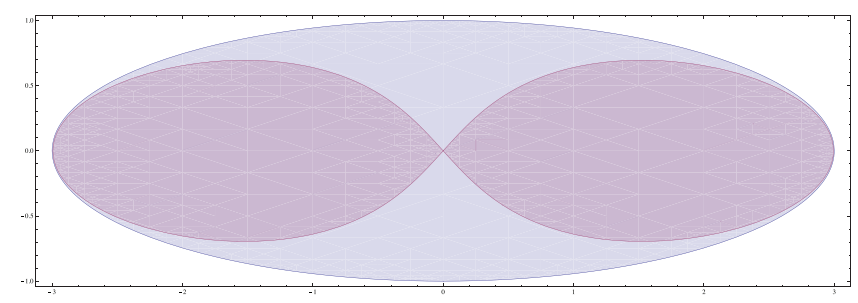

Figure 3. Choices of origin in the dark shaded region give $G_{0}<G_{1}$. 


\section{Existence of homeomorphism $H$ - Proof of Theorem 2.1}

Here we construct a bi-Lipschitz homeomorphism $H: \mathbb{\$} \rightarrow \mathbb{\$}$ with specified Jacobian determinant, as required in Section 2. We apply the construction to ellipsoids in the next section, in order to better understand the geometric factor $G_{1}$.

Write $K(\xi)=R(\xi)^{d} V(\mathbb{B}) / V(\Omega)$ for the desired Jacobian determinant of $H$, which one regards as the mass density for the $H$-pullback of the uniform mass density on the sphere. The following construction of $H$ remains valid whenever $K$ is continuous and positive with $\int_{\Phi} K(\xi) d \xi=|\$|$; the specific form of $K$ is irrelevant.

Two dimensions, $\boldsymbol{d}=2$. In two dimensions we may regard $K$ as a $2 \pi$-periodic function of an angle $\theta$. The Jacobian condition $\mathrm{J} a c_{H}=K$ says $H^{\prime}(\theta)=K(\theta)$, which we satisfy by defining

$$
H(\theta)=\int_{0}^{\theta} K(\omega) d \omega
$$

Note $H$ increases by $2 \pi$ each time $\theta$ increases by $2 \pi$, since $\int_{0}^{2 \pi} K(\omega) d \omega=\left|\$^{1}\right|=$ $2 \pi$. Also, $H^{\prime}=K$ is continuous, and is bounded above and below away from 0 . Hence $H$ defines a $C^{1}$-diffeomorphism of the circle.

Incidentally, this construction shows that $H$ is uniquely determined on the circle, except for post-rotations (adding a constant to $H$ ). We need not consider reflections because $H$ has positive Jacobian and so it must be orientation preserving.

Three dimensions, $\boldsymbol{d}=3$ : the latitude-longitude construction. Let $\left(\theta_{1}, \theta_{2}\right)$ be the standard spherical coordinates, with $0 \leq \theta_{1} \leq \pi$ and $0 \leq \theta_{2} \leq 2 \pi$. We assume $H$ has the form

$$
H\left(\theta_{1}, \theta_{2}\right)=\left(f\left(\theta_{1}\right), g\left(\theta_{1}, \theta_{2}\right)\right),
$$

which means that each line of latitude $\left(\theta_{1}=\right.$ const.) is mapped to another line of latitude, and the longitudinal position is transformed by $g$. We will first determine $f$ by studying how the spacing between lines of latitude must be distorted, and then will determine $g$ by applying the earlier 2-dimensional method on each line of latitude.

Fix the north and south poles (meaning $f(0)=0, f(\pi)=\pi$ ) and require that $g$ increase by $2 \pi$ for each trip around a line of latitude (meaning $g(\cdot, \cdot+2 \pi)-g=2 \pi$ ). Note that

$$
\begin{aligned}
-\left(\cos f\left(\theta_{1}\right)\right)^{\prime} g_{\theta_{2}}\left(\theta_{1}, \theta_{2}\right) & =\left(\sin f\left(\theta_{1}\right)\right) f^{\prime}\left(\theta_{1}\right) g_{\theta_{2}}\left(\theta_{1}, \theta_{2}\right) \\
& =K\left(\theta_{1}, \theta_{2}\right) \sin \theta_{1},
\end{aligned}
$$

by the Jacobian condition $\mathrm{J} a c_{H}=K$. Integrating over $\theta_{2} \in[0,2 \pi]$ gives an equation involving only $\theta_{1}$ :

$$
-\left(\cos f\left(\theta_{1}\right)\right)^{\prime}=\left(\frac{1}{2 \pi} \int_{0}^{2 \pi} K\left(\theta_{1}, \theta_{2}\right) d \theta_{2}\right) \sin \theta_{1} .
$$


This equation can be solved for $\cos f\left(\theta_{1}\right)$ by direct integration, using the north pole condition $\cos f(0)=1$. (The south pole condition $\cos f(\pi)=-1$ then follows automatically, since we have $\int_{0}^{\pi} \int_{0}^{2 \pi} K \sin \theta_{1} d \theta_{2} d \theta_{1}=\int_{\Phi^{2}} K d S=\left|\Phi^{2}\right|=4 \pi$.) Next, we substitute (39) into the left side of (38) to get an equation for $g_{\theta_{2}}$ that can be integrated directly to obtain $g$; we fix the constant of integration by requiring $g\left(\theta_{1}, 0\right)=0$ (which means geometrically that $H$ fixes the prime meridian). One checks easily from (38) and (39) that the construction gives $g\left(\theta_{1}, 2 \pi\right)=2 \pi$ as required.

The above construction guarantees $f^{\prime}>0$ and $g_{\theta_{2}}>0$ away from the poles, and one can check that the resulting $H$ gives a bi-Lipschitz homeomorphism of the sphere.

Remark. The point of this section is to provide a construction of $H$ that can be implemented in practical examples. Many other homeomorphisms also satisfy the Jacobian condition (4). See Dacorogna and Moser [12] for an account of the amazingly varied possibilities.

Higher dimensions. In dimensions 4 and higher, one extends the 3-dimensional construction by means of generalized spherical coordinates. Induction on the dimension provides the analogue of $g$ on lower dimensional "latitudinal spheres". We leave the details to the reader.

\section{Ellipsoidal examples and the geometric factor $G_{1}$}

The homeomorphism $H: \mathbb{\$} \rightarrow \mathbb{S}$ constructed in the preceding section induces a volume-preserving map $T: \Omega \rightarrow \mathbb{B}$, as defined in Section 2. For ellipsoids one could alternatively use the linear map provided by a matrix $M$ with $M(E)=\mathbb{B}$. Which of these two maps will give a better estimate on the eigenvalues in Theorem 3.1? That is, which will give a smaller value for the geometric factor $G_{1}$ ?

For the linear map one has

$$
\begin{aligned}
T(r \xi) & =M(r \xi)=r M \xi, \\
R(\xi) & =\frac{1}{|M \xi|}, \quad H(\xi)=\frac{M \xi}{|M \xi|} .
\end{aligned}
$$

Extending $R$ and $H$ to be homogeneous functions gives

$$
R(x)=\frac{|x|}{|M x|}, \quad H(x)=\frac{M x}{|M x|} .
$$

Somewhat tedious calculations then show that

$$
G_{0}=G_{1}=[V(E) / V(\mathbb{B})]^{2 / d}\|M\|_{H S}^{2} / d .
$$


Thus for ellipsoids we recover our earlier results about linear transformations [33] and [34]. (Those papers treat more general domains than just ellipsoids, of course.)

Let us now compare this "linear" map $T$ with the map $T$ constructed from "spherical coordinates" as in the previous section. Table 1 shows the values of $G_{1}$ in the linear case, and also shows numerical values from the spherical coordinates construction, for various choices of north pole. Note in the table that if the ellipsoid has two equal semi-axes (so that it is a body of revolution), and if we take the remaining axis as the north pole, then we obtain the same value for $G_{1}$ as in the linear case. That equality no longer holds for a generic ellipsoid with unequal semi-axes, and in general the linear construction gives better results than the spherical coordinates one does.

These observations provide some guidance as to how to choose the north pole when constructing $H$ by the spherical coordinates method, for an arbitrary starlike domain.

Table 1. Values of $G_{1}$ for the "linear" construction of $T$, and for various choices of north pole in the "spherical coordinates" construction of $T$.

\begin{tabular}{ccccc}
\hline semiaxes (a,b,c) & linear: $G_{1}=G_{0}$ & North a & North b & North c \\
\hline$(1,1,1)$ & 1 & 1 & 1 & 1 \\
$(1,1,2)$ & 1.19055 & 1.24002 & 1.24002 & 1.19055 \\
$(1,2,2)$ & 1.25992 & 1.25992 & 1.32057 & 1.32057 \\
$(1,2,3)$ & 1.49810 & 1.51620 & 1.73826 & 1.53697 \\
\hline
\end{tabular}

\section{Sloshing problem}

We finish the paper by transferring our results to "sloshing eigenvalues". On a cylinder $C=\Omega \times[-L, 0]$, we consider the following two eigenvalue problems:

$$
\begin{aligned}
\Delta u & =0 & & \text { in } C, \\
u & =0 & & \text { on } \partial C \backslash \Omega, \\
\frac{\partial u}{\partial n} & =\tilde{\lambda} u & & \text { on } \Omega,
\end{aligned}
$$

and

$$
\begin{aligned}
& \Delta u=0 \quad \text { in } C, \\
& \frac{\partial u}{\partial n}=0 \quad \text { on } \partial C \backslash \Omega \text {, } \\
& \frac{\partial u}{\partial n}=\tilde{\mu} u \quad \text { on } \Omega .
\end{aligned}
$$


See Figure 4. The second eigenvalue problem describes frequencies of sloshing of a fluid in the special case of a cylindrical "glass" with uniform cross-sections. (See [15] for a historical review and [5], [20], [26], [27], and [28] for recent developments.)

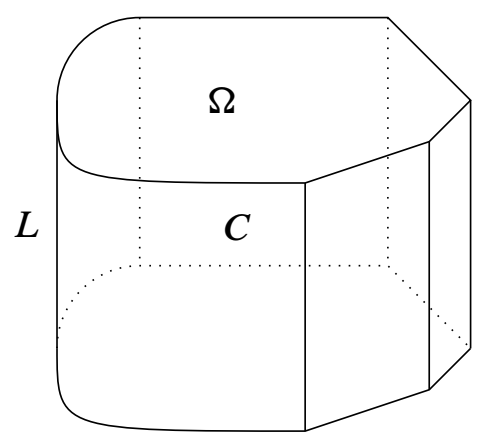

Figure 4. A sloshing cylinder $C$ with height $L$ and starlike cross-section $\Omega$.

When we want to emphasize the dependence on the cylinder depth, we write the eigenvalues as $\tilde{\lambda}_{j}(L)$ and $\tilde{\mu}_{j}(L)$. The eigenvalues are determined by separating the vertical and horizontal variables. One finds

$$
\tilde{\lambda}_{j}(L)=\sqrt{\lambda_{j}} \operatorname{coth}\left(\sqrt{\lambda_{j}} L\right) \text { and } \tilde{\mu}_{j}(L)=\sqrt{\mu_{j}} \tanh \left(\sqrt{\mu_{j}} L\right),
$$

where $\lambda_{j}$ and $\mu_{j}$ are the Dirichlet and Neumann eigenvalues of $\Omega$. The functions $\Phi_{D}(a)=\sqrt{a} \operatorname{coth}(\sqrt{a} L)$ and $\Phi_{N}(a)=\sqrt{a} \tanh (\sqrt{a} L)$ are concave increasing, and so majorization (Appendix A) extends our theorems on Dirichlet and Neumann eigenvalues of the Laplacian to sloshing eigenvalues. The Neumann sloshing conclusion, in 2 dimensions, is that the normalized eigenvalue sum

$$
\sum_{j=2}^{n} \tilde{\mu}_{j}\left(L \sqrt{\frac{A}{G}}\right) \sqrt{\frac{A}{G}}=\sum_{j=2}^{n} \Phi_{N}\left(\mu_{j} A / G\right)
$$

is maximal for the disk, for each $L>0$. (Note here that the depth $L \sqrt{A / G}$ of the cylinder depends on the area and geometric factor of the cross-section.) One can then extend to more general functionals of the $\tilde{\mu}_{j}$, by performing a second majorization.

These methods handle only sloshing in cylindrical glasses, although one can use domain monotonicity (see [5]) to obtain bounds for some other shapes of glass. It would be interesting to prove sharp bounds on eigenvalues of non-cylindrical sloshing regions, by comparing somehow with a domain having rotational symmetry about the vertical axis, as in [26]. 


\section{A. Majorization}

To extend from eigenvalue sums to sums of concave functions of eigenvalues we use the following proposition.

Proposition A.1. Assume $\left\{a_{j}\right\}$ and $\left\{b_{j}\right\}$ are increasing sequences of positive real numbers. Then the following statements are equivalent:

(i) $\sum_{j=1}^{n} a_{j} \leq \sum_{j=1}^{n} b_{j}$ for each $n \geq 1$;

(ii) $\sum_{j=1}^{n} \Phi\left(a_{j}\right) \leq \sum_{j=1}^{n} \Phi\left(b_{j}\right)$ for each $n \geq 1$ and all concave increasing functions $\Phi: \mathbb{R}_{+} \rightarrow \mathbb{R}$.

The result is due to Hardy, Littlewood and Pólya [17], §3.17. They treated decreasing sequences $\left\{a_{j}\right\}$ and $\left\{b_{j}\right\}$ and a convex increasing function $\Phi$, which is equivalent to Proposition A.1 after replacing $\Phi(a)$ with $-\Phi(-a)$. A comprehensive account of majorization methods can be found in the monograph of Marshall, Olkin and Ingram [36]. For equality statements in (i) and (ii), including the infinite series case $n=\infty$, see a paper by Laugesen and Morpurgo [32], Proposition 10.

\section{B. Orbital and spatial averages}

Equality of orbital and spatial averages on the sphere was needed several times in the paper.

\section{Lemma B.1.}

$$
\int_{O(d)} f(U \zeta) d \gamma(U)=\frac{1}{|\mathbb{\$}|} \int_{\mathbb{S}} f\left(\zeta^{\prime}\right) d S\left(\zeta^{\prime}\right)
$$

for any $f \in L^{1}(\$)$ and each $\zeta \in \mathbb{\text { . }}$

Proof of Lemma B.1. The right side of the formula equals

$$
\frac{1}{|\$|} \int_{\Phi} f\left(U \zeta^{\prime}\right) d S\left(\zeta^{\prime}\right)
$$

by a change of variable, for each $U$. Integrating with respect to $U$ gives (by Fubini) that

$$
\frac{1}{|\mathbb{\$}|} \int_{\mathbb{S}} f\left(\zeta^{\prime}\right) d S\left(\zeta^{\prime}\right)=\frac{1}{|\mathbb{\$}|} \int_{\mathbb{S}} \int_{O(d)} f\left(U \zeta^{\prime}\right) d \gamma(U) d S\left(\zeta^{\prime}\right) .
$$

For each $\zeta^{\prime}$ we change variable with $U \mapsto U V$, where $V$ is chosen so that $V \zeta^{\prime}=\zeta$. The lemma follows. 


\section{References}

[1] M. I. Aissen, A set function defined for convex plane domains. Pacific J. Math. 8 (1958), 383-399. MR 0123968 Zbl 0084.18603

[2] M. S. Ashbaugh and R. D. Benguria, A sharp bound for the ratio of the first two eigenvalues of Dirichlet Laplacians and extensions. Ann. of Math. (2) 135 (1992), 601-628. MR 1166646 Zbl 0757.35052

[3] M. S. Ashbaugh and R. D. Benguria, Isoperimetric inequalities for eigenvalues of the Laplacian. In F. Gesztesy, P. Deift, Ch. Galvez, P. Perry, and W Schlag (eds.) Spectral theory and mathematical physics: a Festschrift in honor of Barry Simon's $60^{\text {th }}$ birthday. Quantum field theory, statistical mechanics, and nonrelativistic quantum systems. Papers from the conference held at the California Institute of Technology, Pasadena, CA, March 27-31, 2006. Proceedings of Symposia in Pure Mathematics 76, Part 1. American Mathematical Society, Providence, RI, 2007, 105-139. MR 2310200 MR 2310192 (collection) Zbl 1221.35261 Zbl 1110.00014 (collection)

[4] C. Bandle, Isoperimetric inequalities and applications. Monographs and Studies in Mathematics 7, Pitman (Advanced Publishing Program), Boston, MA, etc., 1980. MR 572958 Zbl 0436.35063

[5] R. Bañuelos, T. Kulczycki, I. Polterovich, and B. Siudeja, Eigenvalue inequalities for mixed Steklov problems. In M. Levitin and D. Vassiliev, Operator theory and its applications. In memory of V. B. Lidskii (1924-2008). American Mathematical Society Translations, Series 2, 231. Advances in the Mathematical Sciences 65. American Mathematical Society, Providence, R.I., 2010, 19-34. MR 2758960 MR 2663905 (collection) Zbl 1217.35127 Zbl 1202.01094 (collection)

[6] M. Bareket, On an isoperimetric inequality for the first eigenvalue of a boundary value problem. SIAM J. Math. Anal. 8 (1977), 280-287. MR 0430552 Zbl 0359.35060

[7] R. D. Benguria and H. Linde Isoperimetric inequalities for eigenvalues of the Laplace operator. In C. Villegas-Blas (ed.), Fourth Summer School in Analysis and Mathematical Physics. Topics in spectral theory and quantum mechanics. Lectures from the school held at the Universidad Nacional Autónoma de México, Cuernavaca, May 2005. Contemporary Mathematics 476. Aportaciones Matemáticas. American Mathematical Society, Providence, R.I., and Sociedad Matemática Mexicana, México, 2008, 1-40. MR 2509107 MR 1500293 (collection) Zbl 1162.35056 Zbl 1152.35001 (collection)

[8] M. van den Berg, On Rayleigh's formula for the first Dirichlet eigenvalue of a radial perturbation of a ball. J. Geom. Anal. 23 (2013), 1427-1440. MR 3078360 Zbl 06203580

[9] M.-H. Bossel, Membranes élastiquement liées: extension du théorème de RayleighFaber-Krahn et de l'inégalité de Cheeger. C. R. Acad. Sci. Paris Sér. I Math. 302 (1986), 47-50. MR 0827106 Zbl 0606.73018

[10] H. J. Brascamp, E. H Lieb, and J. M. Luttinger, A general rearrangement inequality for multiple integrals. J. Funct. Analysis 17 (1974), 227-237. MR 0346109 Zbl 0346109

[11] D. Bucur and A. Giacomini, A variational approach to the isoperimetric inequality for the Robin eigenvalue problem. Arch. Ration. Mech. Anal. 198 (2010), 927-961. MR 2729323 Zbl 1228.49049 
[12] B. Dacorogna and J. Moser, On a partial differential equation involving the Jacobian determinant. Ann. Inst. H. Poincaré Anal. Non Linéaire 7 (1990), 1-26. MR 1046081 Zbl 0707.35041

[13] D. Daners, A Faber-Krahn inequality for Robin problems in any space dimension. Math. Ann. 335 (2006), 767-785. MR 2232016 Zbl 1220.35103

[14] D. Daners and J. B. Kennedy. On the asymptotic behaviour of the eigenvalues of a Robin problem. Differential Integral Equations 23 (2010), 659-669. MR 2654263 Zbl 1240.35370

[15] D. W. Fox and J. R. Kuttler, Sloshing frequencies. Z. Angew. Math. Phys. 34 (1983), 668-696. MR 723140 Zbl 0539.76022

[16] P. Freitas and D. Krejčiřík, A sharp upper bound for the first Dirichlet eigenvalue and the growth of the isoperimetric constant of convex domains. Proc. Amer. Math. Soc. 136 (2008), 2997-3006. MR 2399068 Zbl 1147.58030

[17] G. H. Hardy, J. E. Littlewood, and G. Pólya, Inequalities. Cambridge Mathematical Library, Cambridge University Press, Cambridge, 1988. Reprint of the 1952 edition. MR 944909 Zbl 0634.26008

[18] D. Henry, Perturbation of the boundary in boundary-value problems of partial differential equations. With editorial assistance from J. Hale and A. L. Pereira. London Mathematical Society Lecture Note Series 318, Cambridge University Press, Cambridge, 2005. MR 2160744 Zbl 1170.35300

[19] A. Henrot, Extremum problems for eigenvalues of elliptic operators. Frontiers in Mathematics. Birkhäuser, Basel, 2006. MR 2251558 Zbl 1109.35081

[20] R. A. Ibrahim, Liquid sloshing dynamics. Theory and applications. Cambridge University Press, Cambridge, 2005.

[21] T. Iwaniec and G. Martin, Geometric function theory and non-linear analysis. Oxford Mathematical Monographs, The Clarendon Press, Oxford University Press, New York, 2001. MR 1859913 Zbl 1045.30011

[22] D. Jakobson, M. Levitin, N. Nadirashvili, N. Nigam, and I. Polterovich, How large can the first eigenvalue be on a surface of genus two? Int. Math. Res. Not. 2005 (2005), 3967-3985. MR 2202582 Zbl 1114.58026

[23] B. Kawohl, Rearrangements and convexity of level sets in PDE. Lecture Notes in Mathematics 1150, Springer Verlag, Berlin etc., 1985. MR 0810619 Zbl 0593.35002

[24] S. Kesavan, Symmetrization \& applications. Series in Analysis 3, World Scientific, Hackensack, N.J., 2006. MR 2238193 Zbl 1110.35002

[25] H. Kovařík, On the lowest eigenvalue of Laplace operators with mixed boundary conditions. J. Geom. Anal. 24 (2014), 1509-1525. MR 3223564

[26] T. Kulczycki and M. Kwaśnicki, On high spots of the fundamental sloshing eigenfunctions in axially symmetric domains. Proc. Lond. Math. Soc. (3) 105 (2012), 921-952. MR 2997042 Zbl 1256.35211

[27] T. Kulczycki and N. Kuznetsov, 'High spots' theorems for sloshing problems. Bull. Lond. Math. Soc. 41 (2009), 495-505. MR 2506833 Zbl 1173.35091 
[28] T. Kulczycki and N. Kuznetsov, On the 'high spots' of fundamental sloshing modes in a trough. Proc. R. Soc. Lond. Ser. A Math. Phys. Eng. Sci. 467 (2011), 1491-1502. MR 2782167 Zbl 1219.76007

[29] R. S. Laugesen, Spectral theory of partial differential equations - Lecture notes. Preprint 2012. arXiv:1203.2344 [math.AP]

[30] R. S. Laugesen, Tight frames and rotations: sharp bounds on eigenvalues of the Laplacian. In X. Duong, J. Hogan, Ch. Meaney, and Adam Sikora (eds.), AMSI International Conference on Harmonic Analysis and Applications. Macquarie University, 2011. CMA Proceedings 45. Centre for Mathematics and its Applications Mathematical Sciences Institute, The Australian National University, Camberra, 2013, 63-82.

[31] R. S. Laugesen, J. Liang, and A. Roy, Sums of magnetic eigenvalues are maximal on rotationally symmetric domains. Ann. Henri Poincaré 13 (2012), 731-750. MR 2913619 Zbl 1242.81082

[32] R. S. Laugesen and C. Morpurgo, Extremals for eigenvalues of Laplacians under conformal mapping. J. Funct. Anal. 155 (1998), 64-108. MR 1622804 Zbl 0917.47018

[33] R. S. Laugesen and B. A. Siudeja, Sums of Laplace eigenvalues-rotationally symmetric maximizers in the plane. J. Funct. Anal. 260 (2011), 1795-1823. MR 2754893 Zbl 1215.35112

[34] R. S. Laugesen and B. A. Siudeja, Sums of Laplace eigenvalues: rotations and tight frames in higher dimensions. J. Math. Phys. 52 (2011), Article Id. 093703, 13 pp. MR 2867829 Zbl 1272.35077

[35] J. M. Luttinger, Generalized isoperimetric inequalities. J. Mathematical Phys. 14 (1973), 586-593, 1444-1447, 1448-1450. MR 0337197 MR 0341262

[36] A. W. Marshall and I. Olkin, Inequalities: theory of majorization and its applications. Mathematics in Science and Engineering 143, Academic Press, New York and London, 1979. MR 552278 Zbl 0437.26007

[37] C. Morpurgo, Local extrema of traces of heat kernels on $S^{2}$. J. Funct. Anal. 141 (1996), 335-364. MR 1418510 Zbl 0937.58016

[38] G. Pólya and G. Szegö, Isoperimetric inequalities in mathematical physics. Annals of Mathematics Studies 27. Princeton University Press, Princeton, N.J., 1951. MR 0043486 Zbl 0044.38301

[39] R. Schoen and S.-T Yau, Lectures on differential geometry. Lecture notes prepared by W. Y. Ding, K. Ch. Chang, J. Q. Zhong and Y. Ch. Xu. Translated from the Chinese by W. Y. Ding and S. Y. Cheng. Preface translated from the Chinese by K. Tso. Conference Proceedings and Lecture Notes in Geometry and Topology I. International Press, Cambridge, MA, 1994. MR 1333601 Zbl 0830.53001

[40] J. W. Strutt (Lord Rayleigh), The theory of sound. Volume I. Second edition. Revised and enlarged. Macmillan and Co., London, 1894. JFM 25.1604.01

[41] H. F. Weinberger, An isoperimetric inequality for the $N$-dimensional free membrane problem. J. Rational Mech. Anal. 5 (1956), 633-636. MR 0079286 Zbl 0071.09902 
Received September 20, 2012

Richard S. Laugesen, Department of Mathematics, University of Illinois, Urbana, IL 61801, U.S.A.

E-mail: Laugesen@illinois.edu

Bartłomiej A. Siudeja, Department of Mathematics, University of Oregon, Eugene, OR 97403, U.S.A.

E-mail: Siudeja@uoregon.edu 